\title{
Dynamic structure factor study of diffusion in strongly sheared suspensions
}

\author{
By ALEXANDER M. LESHANSKY AND JOHN F. BRADY \\ Division of Chemistry and Chemical Engineering, California Institute of Technology, \\ Pasadena, CA 91125, USA
}

(Received 30 April 2004 and in revised form 29 September 2004)

Diffusion of neutrally buoyant spherical particles in concentrated monodisperse suspensions under simple shear flow is investigated. We consider the case of nonBrownian particles in Stokes flow, which corresponds to the limits of infinite Péclet number and zero Reynolds number. Using an approach based upon ideas of dynamic light scattering we compute self- and gradient diffusion coefficients in the principal directions normal to the flow numerically from Accelerated Stokesian Dynamics simulations for large systems (up to 2000 particles). For the self-diffusivity, the present approach produces results identical to those reported earlier, obtained by probing the particles' mean-square displacements (Sierou \& Brady, J. Fluid Mech. vol. 506, 2004 p. 285). For the gradient diffusivity, the computed coefficients are in good agreement with the available experimental results. The similarity between diffusion mechanisms in equilibrium suspensions of Brownian particles and in non-equilibrium non-colloidal sheared suspensions suggests an approximate model for the gradient diffusivity: $\boldsymbol{D}^{\nabla} \approx \boldsymbol{D}^{s} / S^{e q}(0)$, where $\boldsymbol{D}^{s}$ is the shear-induced self-diffusivity and $S^{e q}(0)$ is the static structure factor corresponding to the hard-sphere suspension at thermodynamic equilibrium.

\section{Introduction}

The phenomenon of shear-induced diffusion in dispersions has been extensively investigated over the past 30 years. The earliest studies were concerned with the enhanced transport of a passive scalar (dissolved species or a temperature) in a sheared suspension of freely suspended particles (Leal 1973; Nir \& Acrivos 1976). In these studies, it was proposed that the diffusive transport in a surrounding fluid phase can be substantially augmented by micro-convection resulting from the rotation of a particle. In a pioneering experimental study Eckstein, Bailey \& Shapiro (1977) showed that the shear flow causes the particles themselves to exhibit random migrations leading to a diffusive-like behaviour. Subsequently, this intriguing phenomenon of shear-induced diffusion of particles has been studied experimentally (Leighton \& Acrivos 1987; Phillips et al. 1992; Breeveld et al. 1998, 2001), theoretically (Acrivos et al. 1992; da Cunha \& Hinch 1986; Wang, Mauri \& Acrivos 1996, 1998; Morris \& Brady 1996) and computationally (Marchioro \& Acrivos 2001; Drazer et al. 2002; Sierou \& Brady 2004).

The major obstacle to development of a theory of shear-induced diffusion in nonBrownian suspensions at low Reynolds numbers is the fact that the encounter between two perfectly smooth spheres does not lead to a net lateral displacement of either sphere due to the linearity and time-reversibility of the Stokes equations. Thus, at 
the pair level a non-hydrodynamic microscopic mechanism must be introduced to give rise to the microscopic irreversibility that would lead to net displacement upon a single encounter and to a diffusive behaviour upon many successive encounters with neighbouring particles, provided that the motion becomes uncorrelated after a certain time. Following this line, da Cunha \& Hinch (1996) studied the two-particle problem in the presence of small surface roughness, while in Brady \& Morris (1997) residual Brownian motion combined with short-range repulsive forces was the mechanism giving rise to microscale irreversibility. Another possible diffusive mechanism that is of hydrodynamic origin involves interaction of at least three particles (Wang et al. 1996, 1998).

It has been argued by Marchioro \& Acrivos (2001) that although the equations of fluid motion are linear, the equations of particle motion are not, and the complicated hydrodynamic interactions between them mediated by a viscous fluid may lead to the loss of memory in phase space due to chaotic dynamics. Thus, a tracer particle may exhibit a random walk in a homogenous suspension under the action of purely deterministic forces. These arguments are now supported by recent findings of Drazer et al. (2002) who showed via numerical Stokesian Dynamics (SD) simulations on strongly sheared suspensions that the evolution of the system in phase space is indeed chaotic, i.e. the largest Lyapunov exponent is positive. Since in SD simulations a shortrange inter-particle repulsive potential that resembles the hard-sphere interaction is normally used to prevent particles from overlapping, it is not entirely clear whether the origin of the diffusive behaviour is purely hydrodynamic or not. On the other hand, in a related problem of particle sedimentation the chaotic dynamics can be revealed even for three(!) spherical particles settling in a vertical plane in an unbounded viscous fluid at $R e=0$ in the pure hydrodynamic limit (Jánosi et al. 1997).

For colloidal suspensions two relevant particles diffusivities can be identified: (i) the self-diffusivity of a tracer particle in a homogenous suspension; (ii) the gradient diffusivity relating the flux of particles, $\boldsymbol{j}$, to a small steady concentration gradient, $\nabla \phi$ :

$$
\boldsymbol{j}=-\boldsymbol{D}^{\nabla}(\phi) \cdot \nabla \phi .
$$

Note that in contrast to Brownian colloidal dispersions where the motion is thermally driven, there is no short-time diffusivity in the absence of Brownian motion because the initial dynamics of a particle are deterministic. Only at long times such that the shearing motion has forced a particle to encounter many successive collisions with other particles, may the motion become uncorrelated and diffusive. Thus, both the shear-induced self- and gradient diffusivities are 'long-time' diffusivities.

Although self-diffusion coefficients can be calculated using appropriate kinematic descriptions such as the time rate of change of a particle's mean-square displacement or the integral of the velocity autocorrelation function (Marchioro \& Acrivos 2001; Sierou \& Brady 2004), computation of the gradient diffusivity is far from trivial. Marchioro \& Acrivos (2001) proposed a novel method for calculating the gradient diffusivity numerically using Stokesian Dynamics simulations. The essence of their method is that the suspension microstructure relaxes with a rate proportional to the gradient diffusion coefficient. Thus, if the initial microstructure is rather different from that of a suspension that has been steadily sheared for a long time, from probing the rate of relaxation of the initially distorted suspension microstructure towards a steady state, one can extract the gradient diffusivity.

Our approach is different and comes from ideas of dynamic light scattering and concerns the relaxation of the average fluctuation in particle number density rather 
than spatial variations of the macroscopic density itself. As we shall see, the starting point is the so-called dynamic structure factor (or intermediate dynamic scattering function), which allows one to describe both the self- and the gradient diffusivity independently of the underlying microscale dynamics. A recent modification of Stokesian Dynamics, called Accelerated Stokesian Dynamics (ASD) (Sierou \& Brady 2001), is used in simulations. Its low computational cost of $O(N \log N)$, with $N$ being the number of particles, allows us to compute self- and gradient diffusivities from a single long simulation run on steadily sheared homogenous suspensions of $N \sim 10^{2}-$ $10^{3}$ particles without introducing an artificial inhomogeneity to the suspension microstructure. It is shown that the self-diffusivity extracted from the decay of the relevant time autocorrelation is equivalent to the integral of the velocity autocorrelation, which is a well-known kinematic description of the self-diffusivity. The analogous kinematic description of the gradient diffusivity is derived in terms of the velocity crosscorrelation function and the non-equilibrium osmotic compressibility. It is anticipated that this formula should hold in many different physical contexts (e.g. long-time gradient diffusivity in colloidal suspensions) since it is independent of the microscale dynamics and is derived from purely kinematic arguments.

Since diffusion in directions transverse to the direction of the flow is the major mechanism of particle transport, we shall only study diffusion in the directions of the velocity gradient $\left(D_{y y}\right)$ and vorticity $\left(D_{z z}\right)$. Although a diffusivity along the flow direction $\left(D_{x x}\right)$ and an off-diagonal component $\left(D_{x y}\right)$ can also be studied (see Sierou \& Brady 2004), they are negligible compared to the advective transport in those directions and will be not considered here.

The paper is organized as follows. In $\S 2$ we discuss how the diffusion coefficients can be determined using the dynamic structure factor approach. In $\S 3$ we briefly describe the details of the numerical simulations. The results for the diffusivities along both principal directions transverse to the direction of the flow (velocity-gradient and the vorticity directions, respectively) are presented. The computed values of self-diffusion coefficients are reported in $\S 4.1$ and compare favourably with those obtained in Sierou \& Brady (2004) from the mean-square displacement and the velocity autocorrelation. Section 4.2 describes the computation of the gradient diffusion coefficients. Values of gradient diffusion coefficients extrapolated to the limit of an infinite system are compared with available experimental results for a wide range of particle volume fractions. Comparison of our results to the earlier calculations by Marchioro \& Acrivos (2001) is addressed in section $\S 5$. In $\S 6$ a brief summary and concluding remarks are provided.

\section{Dynamic structure factor approach}

To understand how we can determine the diffusivity in shearing flows, consider the following conservation equation for the local particle number density, $n(\boldsymbol{x}, t)$ :

$$
\frac{\partial n}{\partial t}+\dot{\boldsymbol{\Gamma}} \cdot \boldsymbol{x} \cdot \nabla n+\boldsymbol{U} \cdot \nabla n=-\nabla \cdot \boldsymbol{j},
$$

where $\dot{\boldsymbol{\Gamma}}$ is the constant velocity-gradient tensor, $\boldsymbol{U}$ is the bulk average velocity measured at an arbitrary field point, $\boldsymbol{x}_{0}$, from which the bulk shear velocity is referenced and $\boldsymbol{j}$ is the diffusive flux of particles. Neglecting memory effects for time scales over which the diffusion is stationary, the flux should be expressible as a 
generalized Fick's law:

$$
\boldsymbol{j}=-\int \boldsymbol{D}^{c}\left(\boldsymbol{x}-\boldsymbol{x}^{\prime}\right) \cdot \nabla n\left(\boldsymbol{x}^{\prime}, t\right) \mathrm{d} \boldsymbol{x}^{\prime},
$$

where the non-local kernel is identified as the collective diffusivity. The spatial Fourier transform (denoted by ${ }^{\wedge}$ ) of $(2.1)$ is

$$
\frac{\partial \widehat{n}}{\partial t}-\boldsymbol{k} \cdot \dot{\boldsymbol{\Gamma}} \cdot \nabla_{k} \widehat{n}-\mathrm{i} \boldsymbol{k} \cdot \boldsymbol{U} \widehat{n}=-\boldsymbol{k} \cdot \widehat{\boldsymbol{D}}^{c}(\boldsymbol{k}) \cdot \boldsymbol{k} \widehat{n},
$$

which is an analogue of the conservation equation describing the evolution of a passive scalar field in a linear flow with a constant isotropic diffusivity $\boldsymbol{D}=D \boldsymbol{I}$. Note that the spreading or dispersion of the passive scalar due to the shear flow, as characterized by the second moment $\int \boldsymbol{x} \boldsymbol{x} n(\boldsymbol{x}, t) \mathrm{d} \boldsymbol{x}$, may grow faster than linearly in time. This is the well-known Taylor dispersion (Elrick 1962) and is accounted for by the $\boldsymbol{k} \cdot \dot{\boldsymbol{\Gamma}} \cdot \nabla_{k} \widehat{n}$ term on the left-hand side of (2.3).

In a steadily sheared suspension the average density fluctuation is zero and therefore we consider the two-point time autocorrelation of the local particle density, $F(\boldsymbol{k}, t)=\left\langle\widehat{n}(\boldsymbol{k}, t) \widehat{n}^{*}(\boldsymbol{k}, 0)\right\rangle$, where * indicates a complex conjugate and angular brackets denote the ensemble average over all time intervals of duration $t$. Multiplying both sides of (2.3) by $\widehat{n}^{*}$, the evolution equation for $F$ is

$$
\frac{\partial F}{\partial t}-\boldsymbol{k} \cdot \dot{\boldsymbol{\Gamma}} \cdot \nabla_{k} F-\mathrm{i} \boldsymbol{k} \cdot \boldsymbol{U} F+\left\langle\widehat{n} \boldsymbol{k} \cdot \dot{\boldsymbol{\Gamma}} \cdot \nabla_{k} \widehat{n}^{*}\right\rangle=-\boldsymbol{k} \cdot \widehat{\boldsymbol{D}}^{c}(\boldsymbol{k}) \cdot \boldsymbol{k} F .
$$

The above equations for $\widehat{n}$ and $F$ have the expected form for a diffusive process in a linear flow, and, by comparison to the equation derived from the particle dynamics, can be used to obtain the proper expressions for the diffusivities.

The local particle number density at any point $\boldsymbol{x}$ can be represented in terms of distributions

$$
n(x, t)=\sum_{\alpha=1}^{N} \delta\left(x-x_{\alpha}(t)\right),
$$

where $\boldsymbol{x}_{\alpha}(t)$ is the position of particle $\alpha$ at time $t$. Thus, the spatial Fourier transform of the particle number density is

$$
\widehat{n}(\boldsymbol{k}, t)=\int \exp [\mathrm{i} \boldsymbol{k} \cdot \boldsymbol{x}] \sum_{\alpha=1}^{N} \delta\left(\boldsymbol{x}-\boldsymbol{x}_{\alpha}(t)\right) \mathrm{d} \boldsymbol{x}=\sum_{\alpha=1}^{N} \exp \left[\mathrm{i} \boldsymbol{k} \cdot \boldsymbol{x}_{\alpha}(t)\right],
$$

and the autocorrelation in number density becomes

$$
F(\boldsymbol{k}, t)=\frac{1}{N}\left\langle\widehat{n}(\boldsymbol{k}, t) \widehat{n}^{*}(\boldsymbol{k}, 0)\right\rangle=\frac{1}{N}\left\langle\sum_{\alpha, \beta}^{N} \exp \left[\mathrm{i} \boldsymbol{k} \cdot\left(\boldsymbol{x}_{\alpha}(t)-\boldsymbol{x}_{\beta}(0)\right)\right]\right\rangle,
$$

where angular brackets \langle\rangle denote the appropriate ensemble average. It it readily seen that as the constant background level of $n$ is irrelevant, $F$ is the autocorrelation of the density fluctuations. In dynamic light scattering $F$ is known as the dynamic structure factor (also known as the intermediate scattering function) and can be probed via the time correlation of the intensity of the scattered electric field (Berne \& Pecora 1976; Pusey 1991). The self-dynamic structure factor (a.k.a self-intermediate scattering function) can be introduced in an analogous way by neglecting cross-correlations in 
positions of different particles in (2.7),

$$
F_{s}(\boldsymbol{k}, t)=\frac{1}{N}\left\langle\sum_{\alpha=1}^{N} \exp \left[\mathrm{i} \boldsymbol{k} \cdot\left(\boldsymbol{x}_{\alpha}(t)-\boldsymbol{x}_{\alpha}(0)\right)\right]\right\rangle .
$$

The self-diffusive motion can be probed via $F$ if some small portion of the particles in a sampling volume are tagged (e.g. have different refractive index). Indeed, due to the diluteness of the tagged particles the phase factor $\left|\mathrm{i} \boldsymbol{k} \cdot\left(\boldsymbol{x}_{\alpha}(t)-\boldsymbol{x}_{\beta}(0)\right)\right|$ is large even for small $k$ and thus the cross-terms $\alpha \neq \beta$ in $F$ involving correlation in relative positions of different particles disappear upon averaging and $F$ becomes the selfdynamic structure factor, $F_{s}$. On the other hand, at large values of $k$ small variations in relative particle positions, $\left(\boldsymbol{x}_{\alpha}(t)-\boldsymbol{x}_{\beta}(0)\right)$, cause large variations in the phase factors and, again, $F$ tends to $F_{s}$, regardless of the labelling of individual particles. Therefore, in the limit $k a \gg 1, F$ also measures the average self-motion of individual particles. Here $a$ is the size of a particle. In general, at moderate $k$, the cross-terms involving $\left(\boldsymbol{x}_{\alpha}(t)-\boldsymbol{x}_{\beta}(0)\right)$ are important and $F$ is related to the collective motion of particles (Pusey 1991). In computer simulations both $F$ and $F_{s}$ can be probed directly from the exact knowledge of the particles' positions as a function of time.

Next we differentiate $F$ with respect to time to obtain

$$
\dot{F}(\boldsymbol{k}, t)=\mathrm{i} \boldsymbol{k} \cdot \frac{1}{N}\left\langle\sum_{\alpha=1}^{N} \boldsymbol{U}_{\alpha} \exp \left[\mathrm{i} \boldsymbol{k} \cdot\left(\boldsymbol{x}_{\alpha}(t)-\boldsymbol{x}_{\beta}(0)\right)\right]\right\rangle,
$$

where $\boldsymbol{U}_{\alpha}$ denotes the velocity of the particle $\alpha$ at time $t$. We write $\boldsymbol{U}_{\alpha}$ as

$$
\begin{aligned}
\boldsymbol{U}_{\alpha} & =\dot{\boldsymbol{\Gamma}} \cdot\left(\boldsymbol{x}_{\alpha}-\boldsymbol{x}_{0}\right)+\boldsymbol{U}^{\infty}\left(\boldsymbol{x}_{0}\right)+\boldsymbol{U}_{\alpha}^{\prime} \\
& =\dot{\boldsymbol{\Gamma}} \cdot \boldsymbol{x}_{\alpha}+\boldsymbol{U}^{*}+\boldsymbol{U}_{\alpha}^{\prime},
\end{aligned}
$$

where $\dot{\boldsymbol{\Gamma}}$ is the velocity-gradient tensor, $\boldsymbol{U}^{\infty}$ is the bulk average velocity measured at the arbitrary field point, $\boldsymbol{x}_{0}$, from which the bulk shear velocity is referenced and $\boldsymbol{U}_{\alpha}^{\prime}$ is the configuration-dependent velocity fluctuation of particle $\alpha$ - that is, the velocity of particle $\alpha$ relative to the uniform and bulk shearing motions. Here, $\boldsymbol{U}^{*}=\boldsymbol{U}^{\infty}-\dot{\boldsymbol{\Gamma}} \cdot \boldsymbol{x}_{0}$. Upon inserting $\boldsymbol{U}_{\alpha}$ from (2.10) in (2.9) we obtain

$$
\begin{aligned}
\dot{F}-\boldsymbol{k} \cdot \dot{\boldsymbol{\Gamma}} \cdot \nabla_{k} F-\mathrm{i} \boldsymbol{k} \cdot \boldsymbol{U}^{*} F & -\mathrm{i} \boldsymbol{k} \cdot \dot{\boldsymbol{\Gamma}} \cdot \frac{1}{N}\left\langle\sum_{\alpha, \beta}^{N} \boldsymbol{x}_{\beta}(0) \exp \left[\mathrm{i} \boldsymbol{k} \cdot\left(\boldsymbol{x}_{\alpha}(t)-\boldsymbol{x}_{\beta}(0)\right]\right\rangle\right. \\
& =\mathrm{i} \boldsymbol{k} \cdot \frac{1}{N}\left\langle\sum_{\alpha, \beta}^{N} \boldsymbol{U}_{\alpha}^{\prime} \exp \left[\mathrm{i} \boldsymbol{k} \cdot\left(\boldsymbol{x}_{\alpha}(t)-\boldsymbol{x}_{\beta}(0)\right)\right]\right\rangle \cdot
\end{aligned}
$$

It is straightforward to show that the last term on the left-hand side of (2.11) is equal to the term $\left\langle\widehat{n} \boldsymbol{k} \cdot \dot{\boldsymbol{\Gamma}} \cdot \nabla_{k} \widehat{n}^{*}\right\rangle$ in (2.4). Moreover, it follows from symmetry that

$$
\frac{1}{N}\left\langle\sum_{\alpha, \beta}^{N}\left(\boldsymbol{x}_{\beta}(0)-\boldsymbol{x}_{c m}\right) \exp \left[\mathrm{i} \boldsymbol{k} \cdot\left(\boldsymbol{x}_{\alpha}(t)-\boldsymbol{x}_{\beta}(0)\right]\right\rangle=0\right.
$$

where $\boldsymbol{x}_{c m}$ is the position of the centre of mass of the $N$-particle configuration at $t=0$. Therefore, (2.11) can be re-written as

$$
\dot{F}-\boldsymbol{k} \cdot \dot{\boldsymbol{\Gamma}} \cdot \nabla_{k} F-\mathrm{i} \boldsymbol{k} \cdot \widetilde{\boldsymbol{U}}^{*} F=\mathrm{i} \boldsymbol{k} \cdot \frac{1}{N}\left\langle\sum_{\alpha, \beta}^{N} \boldsymbol{U}_{\alpha}^{\prime} \exp \left[\mathrm{i} \boldsymbol{k} \cdot\left(\boldsymbol{x}_{\alpha}(t)-\boldsymbol{x}_{\beta}(0)\right)\right\rangle\right.
$$


with

$$
\widetilde{\boldsymbol{U}}^{*}=\boldsymbol{U}^{\infty}-\dot{\boldsymbol{\Gamma}} \cdot\left(\boldsymbol{x}_{0}-\boldsymbol{x}_{c m}\right)
$$

indicating that the natural origin in a homogeneous suspension is the centre of mass of the particles. Similarly, the term $\left\langle\widehat{n} \boldsymbol{k} \cdot \dot{\boldsymbol{\Gamma}} \cdot \nabla_{k} \widehat{n}^{*}\right\rangle$ in (2.3) can be shown to be zero.

Thus, comparing (2.12) and (2.4) we identify the collective diffusivity via the following ansatz:

$$
\mathrm{i} \boldsymbol{k} \cdot \frac{1}{N}\left\langle\sum_{\alpha, \beta}^{N} \boldsymbol{U}_{\alpha}^{\prime} \exp \left[\mathrm{i} \boldsymbol{k} \cdot\left(\boldsymbol{x}_{\alpha}(t)-\boldsymbol{x}_{\beta}(0)\right)\right]\right\rangle=-\boldsymbol{k} \cdot \widehat{\boldsymbol{D}}^{c}(\boldsymbol{k}) \cdot \boldsymbol{k} F,
$$

where $\widehat{\boldsymbol{D}}^{c}(\boldsymbol{k})$ is generally $k$-dependent as the process depends on the spatial scale of the density fluctuation. As defined, the collective diffusivity is also, in general, time dependent, while it is expected to become stationary after a finite correlation time.

When $k a \ll 1$ the scale of the density fluctuation is much larger than the size of a single particle, and the relaxation of the long-wavelength density fluctuation yields a particle flux which is the same as if a small constant density gradient persisted everywhere (Rallison \& Hinch 1986). Therefore, as $k a \rightarrow 0$, we expect that $\widehat{\boldsymbol{D}}^{c}(\boldsymbol{k})$ will asymptote to the constant gradient diffusivity, $\widehat{\boldsymbol{D}}^{\nabla}$.

When self-diffusion is considered, neglecting the cross-terms involving correlation between positions of distinct particles will result in the same equation as (2.11) with $F$ becoming $F_{s}$ and the self-diffusivity can be defined via

$$
\mathrm{i} \boldsymbol{k} \cdot \frac{1}{N}\left\langle\sum_{\alpha=1}^{N} \boldsymbol{U}_{\alpha}^{\prime} \exp \left[\mathrm{i} \boldsymbol{k} \cdot\left(\boldsymbol{x}_{\alpha}(t)-\boldsymbol{x}_{\alpha}(0)\right)\right]\right\rangle=-\boldsymbol{k} \cdot \widehat{\boldsymbol{D}}^{s} \cdot \boldsymbol{k} F_{s} .
$$

At long times, tagged particles wander far compared to their size to create a diffusive motion, but still a small distance compared to the lengthscale of the spatial variation of the tracer particles, $k^{-1}$. Thus, in the limit $k a \rightarrow 0, \boldsymbol{D}^{s}$ in (2.14) represents the longtime particle self-diffusivity. The self-diffusivity $\boldsymbol{D}^{s}$ is anticipated to be independent of $k$.

Note that for $\boldsymbol{k} \perp \dot{\boldsymbol{\Gamma}}, \boldsymbol{U}^{*}$ the convective terms on the left-hand side of (2.11) drop out yielding the familiar expressions

$$
-\frac{\dot{F}}{k^{2} F}=\tilde{\boldsymbol{k}} \cdot \widehat{\boldsymbol{D}}_{\perp}^{c}(\boldsymbol{k}) \cdot \tilde{\boldsymbol{k}}, \quad-\frac{\dot{F}_{s}}{k^{2} F_{s}}=\tilde{\boldsymbol{k}} \cdot \widehat{\boldsymbol{D}}_{\perp}^{s} \cdot \tilde{\boldsymbol{k}}
$$

Here the overdot denotes the time derivative and $\tilde{\boldsymbol{k}}=\boldsymbol{k} /|\boldsymbol{k}|$. The temporal behaviour of $F$ and $F_{s}$ in (2.15) is well known in equilibrium colloidal suspensions in the absence of an imposed flow $\left(\dot{\boldsymbol{\Gamma}}=\boldsymbol{U}^{*}=0\right)$ (Berne \& Pecora 1976), with scalar diffusivities $\tilde{\boldsymbol{k}} \cdot \widehat{\boldsymbol{D}}^{s, c} \cdot \tilde{\boldsymbol{k}}=D^{s, c}$ due to rotational invariance. Our derivation (2.12)-(2.15) gives the appropriate generalization necessary for linear flows and provides the operational means to determine the diffusivities from the decay of the dynamic structure factor.

In the small- $k$ limit the left-hand side of $(2.14)$ becomes $-\boldsymbol{k} \cdot N^{-1}\left\langle\sum_{\alpha=1}^{N} \boldsymbol{U}_{\alpha}^{\prime}\right.$ $\left.\Delta \boldsymbol{x}_{\alpha}(t)\right\rangle \cdot \boldsymbol{k}$ since $\left\langle\sum_{\alpha} \boldsymbol{U}_{\alpha}^{\prime}\right\rangle=0, F_{s} \sim 1$, and therefore

$$
\widehat{\boldsymbol{D}}^{s}=\frac{1}{N}\left\langle\sum_{\alpha=1}^{N} \int_{\tau=0}^{t} \boldsymbol{U}_{\alpha}^{\prime}(t) \boldsymbol{U}_{\alpha}(\tau) \mathrm{d} \tau\right\rangle .
$$

The self-diffusivity in a linear flow can be expressed in terms of autocorrelation between the velocity fluctuation and the actual velocity $\boldsymbol{U}_{\alpha}$ in accord with findings of 
Sierou \& Brady (2004), who derived (2.16) from purely kinematic arguments. With a finite correlation time the integral in (2.16) results in a stationary diffusivity $\widehat{\boldsymbol{D}}^{s}$ (Marchioro \& Acrivos 2001; Sierou \& Brady 2004). In the absence of the imposed flow (2.16) becomes the well-known expression for the equilibrium self-diffusivity in terms of the integral over the velocity autocorrelation.

For the collective diffusivity as defined by (2.13), expanding the phase factor on the left-hand side near $k=0$ as

$\exp \left[\mathrm{i} \boldsymbol{k} \cdot\left(\boldsymbol{x}_{\alpha}(t)-\boldsymbol{x}_{\beta}(0)\right)\right]=\exp \left[\mathrm{i} \boldsymbol{k} \cdot\left(\boldsymbol{x}_{\alpha}(t)-\boldsymbol{x}_{\beta}(t)\right)\right]\left[1+\mathrm{i} \boldsymbol{k} \cdot \Delta \boldsymbol{x}_{\beta}-\frac{1}{2} \boldsymbol{k} \boldsymbol{k}: \Delta \boldsymbol{x}_{\beta} \Delta \boldsymbol{x}_{\beta}+\ldots\right]$,

with $\Delta \boldsymbol{x}_{\beta}=\boldsymbol{x}_{\beta}(t)-\boldsymbol{x}_{\beta}(0)$, one arrives at

$$
\widehat{\boldsymbol{D}}^{c}=F^{-1} \frac{1}{N}\left\langle\sum_{\alpha, \beta}^{N}\left(\boldsymbol{U}_{\alpha}^{\prime} \Delta \boldsymbol{x}_{\beta}+\frac{1}{2}\left(\mathrm{i} \boldsymbol{k} \cdot \Delta \boldsymbol{x}_{\beta}\right) \boldsymbol{U}_{\alpha}^{\prime} \Delta \boldsymbol{x}_{\beta}-\ldots\right) \exp \left[\mathrm{i} \boldsymbol{k} \cdot\left(\boldsymbol{x}_{\alpha}(t)-\boldsymbol{x}_{\beta}(t)\right)\right]\right\rangle \text {. }
$$

Also, using (2.17) it can be shown that in the small- $k$ limit the dynamic structure factor, $F$, asymptotes to a static structure factor, $S(\boldsymbol{k})$, that probes the instantaneous microstructure of the suspension at wavevector $\boldsymbol{k}$ :

$$
F(\boldsymbol{k}, t) \sim S(\boldsymbol{k})=\frac{1}{N}\left\langle\sum_{\alpha, \beta}^{N} \exp \left[\mathrm{i} \boldsymbol{k} \cdot\left(\boldsymbol{x}_{\alpha}(t)-\boldsymbol{x}_{\beta}(t)\right)\right]\right\rangle=\frac{1}{N}\left\langle\left|\sum_{\alpha=1}^{N} \exp \left[\mathrm{i} \boldsymbol{k} \cdot \boldsymbol{x}_{\alpha}\right]\right|^{2}\right\rangle,
$$

which is anticipated to be stationary for a steadily sheared suspension. Thus, the leading-order term of the small- $k$ expansion of $\widehat{\boldsymbol{D}}^{c}$ in (2.18) gives the gradient diffusivity that can be expressed in terms of the velocity cross-correlation,

$$
\widehat{\boldsymbol{D}}^{\nabla}=\lim _{k \rightarrow 0} \frac{1}{N S(\boldsymbol{k})}\left\langle\sum_{\alpha, \beta}^{N} \mathscr{M}_{\alpha \beta} \exp \left[\mathrm{i} \boldsymbol{k} \cdot\left(\boldsymbol{x}_{\alpha}(t)-\boldsymbol{x}_{\beta}(t)\right)\right]\right\rangle=\lim _{k \rightarrow 0} \frac{\mathscr{H}(\boldsymbol{k}, t)}{S(\boldsymbol{k})},
$$

where

$$
\mathscr{M}_{\alpha \beta}=\int_{\tau=0}^{t} \boldsymbol{U}_{\alpha}^{\prime}(t) \boldsymbol{U}_{\beta}(\tau) \mathrm{d} \tau .
$$

As for the self-diffusivity, in the long-time limit the correlation is lost and the integral over the velocity cross-correlation is stationary. Also note that one cannot simply pass to the limit by setting $\boldsymbol{k}=\mathbf{0}$ in the exponential factor that multiplies $\mathscr{M}_{\alpha \beta}$ in (2.20) since the doubly infinite $\operatorname{sum}\left\langle\sum_{\alpha \beta} \mathscr{M}_{\alpha \beta}\right\rangle=0$ due to the fact that the mean of the velocity fluctuation is zero.

An expression analogous to (2.20) for the short-time collective diffusivity in an equilibrium colloidal dispersion valid for an arbitrary $k$ was derived by Pusey (1991) using a short-time expansion of $F$. Indeed, in the short-time Brownian regime, i.e. $a^{2} / v \ll t \ll a^{2} / D_{o}$, where $D_{o}$ is the Brownian diffusivity of an isolated particle, the colloidal particles have hardly moved from their initial positions, $\left|\boldsymbol{k} \cdot \Delta \boldsymbol{x}_{\beta}\right| \ll 1$ holds even for large values of $k$ and (2.20) is expected to be valid for a wide range of wavevectors. Segré, Behrend \& Pusey (1995) used (2.20) to evaluate the short-time collective diffusivity from lattice Boltzmann simulations of an equilibrium colloidal dispersion. Since for times $t \ll a^{2} / D_{o}$ (2.20) is asymptotically valid for arbitrary $k$, it can be viewed as the effective $k$-dependent diffusivity that asymptotes to the shorttime gradient diffusivity and to the short-time self-diffusivity in the low- and high- $k$ limits, respectively (Russel \& Glendinning 1981). Indeed, for $k a \gg 1, \mathscr{H} \rightarrow \widehat{\boldsymbol{D}}^{s}$ because the contribution from the cross-terms vanishes on average due to large variations in 
the phase factor $\left|\mathrm{i} \boldsymbol{k} \cdot\left(\boldsymbol{x}_{\alpha}-\boldsymbol{x}_{\beta}\right)\right|$ even for small instantaneous relative separations, while $S^{e q}(k) \rightarrow 1$ as $k \rightarrow \infty$. In contrast to the equilibrium case, for shear-induced diffusion, (2.20) cannot be viewed as the $k$-dependent effective diffusivity for a wide range of $k$ since the motion is not diffusive for short times $t \ll \dot{\gamma}^{-1}$, with $\dot{\gamma}$ being the shear rate. Therefore, (2.20) is only asymptotically valid in the low- $k$ limit.

For equilibrium colloidal suspensions there is a simple physical interpretation of the collective diffusivity, (2.20). In the limit of small $k$, it is well known that the magnitude of the density fluctuations of a system, as characterized by the static structure factor $S(0)$, is related to its isothermal compressibility $(\partial n / \partial \Pi)_{T}$ (Hansen \& McDonald 1986; Pusey 1991):

$$
S^{e q}(0)=k_{B} T\left(\frac{\partial n}{\partial \Pi}\right)_{T} .
$$

Thus, $S^{e q}(0)^{-1}$ can be interpreted as a thermodynamic force associated with the density gradient which drives the diffusion, and the hydrodynamic factor, $\mathscr{H}(k)$, in (2.20) can be interpreted as the collective mobility or the mean sedimentation velocity relative to zero-volume-flux axes (Batchelor 1976). For a dilute colloidal suspension of hard spheres $S^{e q}(0) \sim 1-8 \phi$ due to excluded volume effects, while the collective mobility is given by the average sedimentation velocity, $\mathscr{H}(0) \sim(6 \pi \eta a)^{-1}(1-6.55 \phi)$, leading to the classical result $D^{\nabla}=k_{B} T \mathscr{H}(0) / S^{e q}(0) \sim D_{o}(1+1.45 \phi)$ (Batchelor 1976).

For non-colloidal sheared suspensions, $S(\boldsymbol{k})$, which is a mathematical construct, can be interpreted as the inverse of the non-equilibrium osmotic compressibility that results from the shear-induced hydrodynamic (and interparticle) forces among particles and (2.20) for $\widehat{\boldsymbol{D}}^{c}$ can be given a similar interpretation.

Note that the expressions derived for both the self- (2.16) and gradient (2.20) diffusivities are independent of the details of the microscale dynamics and should hold quite generally, and specifically, for the case of shear-induced diffusion of non-colloidal particles far from thermodynamic equilibrium. The only proviso is that the correlation time must be finite so that a constant diffusivity is attained in the long-time limit.

\section{The simulation method}

In this study the recent modification of the conventional Stokesian Dynamics (SD) method (Bossis \& Brady 1984), so-called Accelerated Stokesian Dynamics (ASD) is used. The detailed description of ASD is provided in Sierou \& Brady (2001). Without going into the details of the new implementation, the major advantage of ASD is its high computational efficiency that scales as $O(N \log N)$ vs. $O\left(N^{3}\right)$ for SD, with $N$ being the number of particle in the simulation box. This significant improvement allows large systems of the order of $10^{3}$ particles to be simulated, an ability that will be shown to be critical for reliable extrapolation to infinite system size for the gradient diffusivity.

The simple shear flow of a monodisperse suspension of non-Brownian spherical particles with radii $a$ in a viscous fluid of viscosity $\eta$ is considered. Non-Brownian implies that the Péclet number, $P e=a^{2} \dot{\gamma} / 2 D_{o}$, is infinitely large. Here, $\dot{\gamma}$, is a shear rate and $D_{o}=k T / 6 \pi \eta a$ is the Brownian diffusivity of an isolated particle. We also assume Stokes flow conditions such that the particle Reynolds number, $\operatorname{Re}_{p}=a^{2} \dot{\gamma} / \nu$, where $v$ is the kinematic viscosity of the fluid, is vanishingly small. Also, following previous numerical studies a short-range repulsive interparticle force was introduced to prevent particles from overlapping. We used the same form of the pair-wise interparticle force 


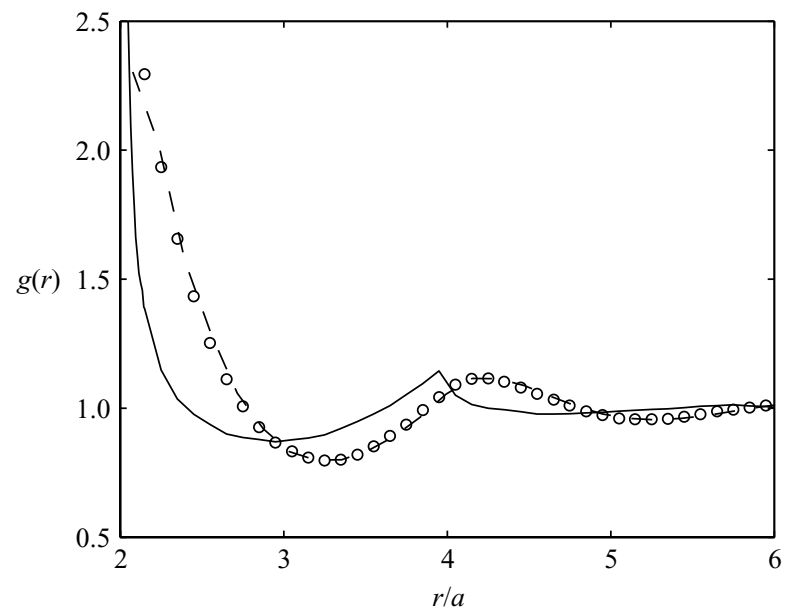

FIGURE 1. Radial pair-distribution function $g(r)$ for $\phi=0.35$. The dashed line is the PercusYevic closure for a hard-sphere suspension at equilibrium, open circles $(O)$ are the results of Brownian dynamics simulations, and the solid line corresponds to the angularly averaged value $\langle g(\boldsymbol{r})\rangle_{\Omega}$ for a non-colloidal suspension of hard spheres in a simple shear flow $(N=512)$ corresponding to infinite Péclet number.

as in Sierou \& Brady (2004), where the self-diffusivity was investigated:

$$
\boldsymbol{F}_{\alpha \beta}=F_{0} \frac{\tau \mathrm{e}^{-\tau \epsilon}}{1-\mathrm{e}^{-\tau \epsilon}} \boldsymbol{e}_{\alpha \beta},
$$

where $6 \pi a^{2} \dot{\gamma} \boldsymbol{F}_{\alpha \beta}$ is the dimensional force exerted on sphere $\alpha$ by sphere $\beta, F_{0}$ stands for the dimensionless magnitude of the force, $\tau$ relates to its range, $\epsilon=(r-2 a) / a$ is the dimensionless separation distance between particles' surfaces, and $\boldsymbol{e}_{\alpha \beta}$ is the unit director connecting the centres of the two particles. In the present investigation the value of $\tau F_{0}$ was set to unity, while $\tau$ was set at 1000 . To demonstrate that this particular form of the interparticle force does not introduce any significant disturbance to the suspension microstructure at equilibrium, we pre-equilibrated 500 particles using standard Brownian dynamics (BD) simulations and compared the resulting pair-distribution function $g(r)$ with the Percus-Yevic (PY) closure for hard spheres (Hansen \& McDonald 1986). As seen in figure 1, the results of the BD simulations (open circles) and the PY closure (the dashed line) are in very close agreement. The considerable difference between the angularly averaged $\langle g(\boldsymbol{r})\rangle_{\Omega}$ corresponding to a strongly sheared non-colloidal suspension evaluated from the ASD simulation (the solid line) and an equilibrium $g(r)$ (the dashed line) indicates the strong effect of the shear on the suspension microstructure (e.g. Gadala-Maria \& Acrivos 1980; Brady \& Morris 1997; Sierou \& Brady 2002). We shall see in $\S 4$ that the shear-induced distortion of the suspension microstructure occurs not only in the direction of the flow, but also in the plane normal to the flow.

Throughout the paper we use non-dimensional lengths scaled with the particle radius $a$ and non-dimensional times scaled with $\dot{\gamma}^{-1}$. Thus, the diffusivities are scaled by $a^{2} \dot{\gamma}$, wavevectors by $a^{-1}$ and velocities by $a \dot{\gamma}$. A fourth-order Adams-Bashforth time-integration scheme was used, while simulation time steps ranged from $\Delta t=$ $5 \times 10^{-3}$ to $1 \times 10^{-4}$ depending on the particle volume fraction. Random initial hardsphere configurations are created using standard methods of molecular dynamics. 

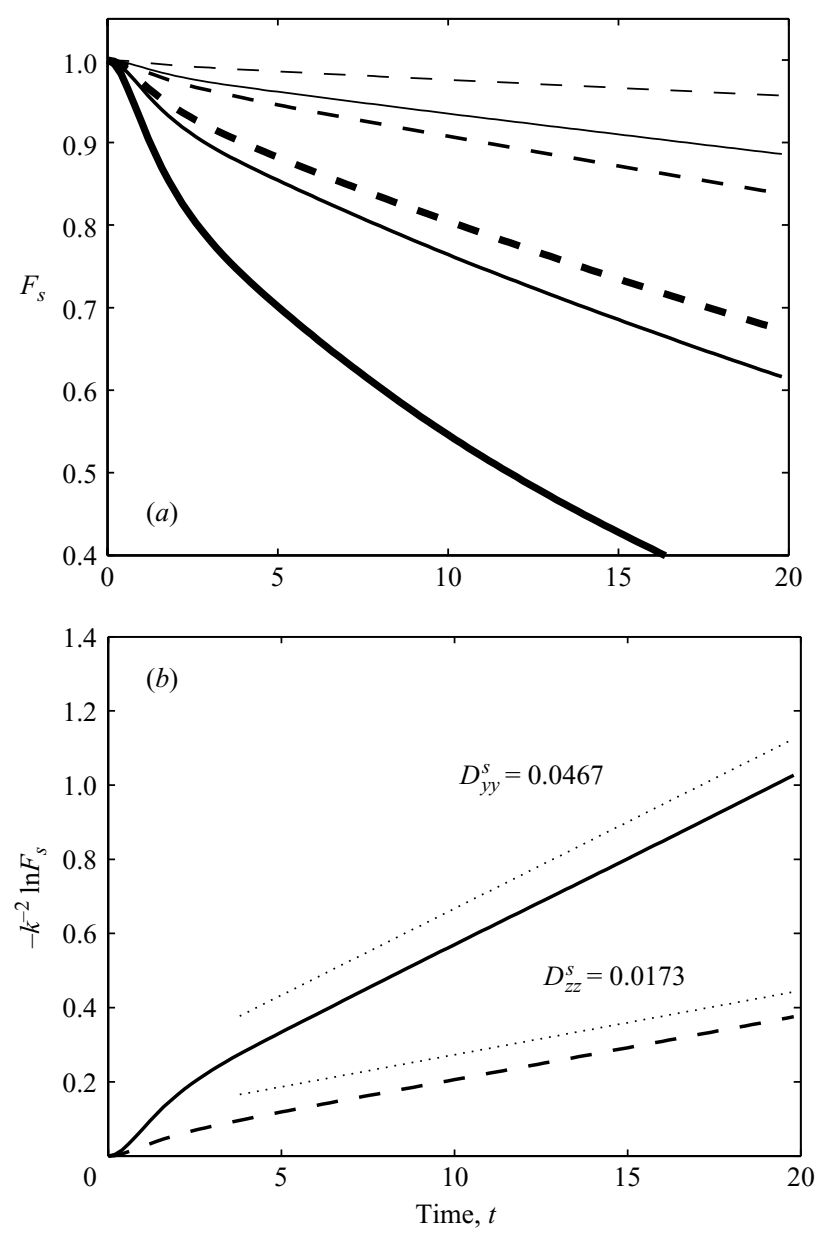

FIGURE 2. Decay of the self-dynamic structure factor, $F_{s}(\boldsymbol{k}, t)$, vs. dimensionless time, $t$, for a system of $N=512, \phi=0.35$ over a total strain of 20. (a) Solid lines correspond to wavevectors $\boldsymbol{k}=2 \pi m / H \boldsymbol{e}_{y}$ and dashed lines to $\boldsymbol{k}=2 \pi m / H \boldsymbol{e}_{z}$ with $m=1,2,3$ (thicker lines correspond to larger $k$ ). (b) The dependence $-k^{-2} \ln F_{s}(\boldsymbol{k}, t)$ vs. $t:-, \boldsymbol{k}=2 \pi m / H \boldsymbol{e}_{y} ;---, \boldsymbol{k}=2 \pi m / H \boldsymbol{e}_{z}$ with $m=1,2,3$. Curves corresponding to different wavevector in both $y$ and $z$ directions collapse onto single curves, resulting in $k$-independent self-diffusivities in the long-time limit. The resulting values of the self-diffusion coefficients are found as the slopes of the curves.

\section{Results}

\subsection{Transverse self-diffusivity}

First we tested the proposed approach by calculating self-diffusion coefficients, $D_{y y}^{s}$ and $D_{z z}^{s}$ (where $ح$ is ommited for simplicity) for a system of $N=512$ particles at a volume fraction $\phi=0.35$. Figure 2 shows the decay of the self-dynamic structure factor, $F_{s}(\boldsymbol{k}, t)$, calculated using (2.8) for three different values of the wavevector $k=2 \pi \mathrm{m} / \mathrm{H}$, where $m=1,2,3$ in both directions $y$ and $z$ for strains up to 20. Here, $H$ is the height of the simulation cell scaled with the particle radius $a$. To improve the statistics we averaged the data over several simulation runs each of 200 time strains starting from independent random initial realizations. Following Sierou \& Brady (2004) to further reduce the statistical noise, the values of $F_{s}$ are averaged over all possible (overlapping) time intervals available for each simulation run. It is evident from figure $2(a)$ that 


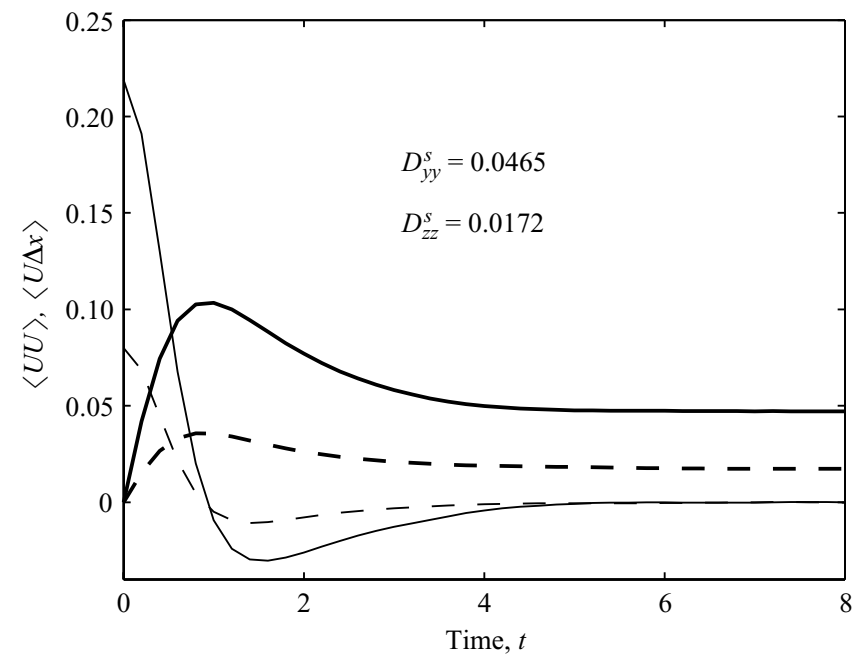

FiguRE 3. The velocity auto-correlation functions and their integrals for a system of $N=512$, $\phi=0.35$. The solid lines correspond to the self-diffusivity in the velocity-gradient direction, while the dashed lines correspond to the vorticity direction. Thinner lines are the velocity auto-correlation functions, while thicker lines are their integrals. The resulting values of the self-diffusivities are given by the areas under the curves or by the plateau values of the integral lines.

after a short time of $\sim 5$ strains the linear diffusive behavior is established and $F_{s}$ decays exponentially according to (2.15). When $-k^{-2} \ln F_{s}$ is plotted as a function of time (see figure $2 b$ ), the curves corresponding to different $k$ in figure 2( $a$ ) collapse onto one curve resulting in a $k$-independent self-diffusivity as anticipated from (2.16). The fact that the same value of $\boldsymbol{D}^{s}$ is recovered not only for small $k$ but even for $k \approx 1$ reflects the fact that, unlike light scattering experiments, in simulations we can 'tag' all the particles in the cell and probe $F_{s}$ at arbitrary $\boldsymbol{k}$ without being concerned about correlations between distinct particles. The self-diffusion coefficients shown in the figure $2(b)$ are found as the slopes of the curves. For comparison, self-diffusion coefficients are evaluated from the integral of the velocity auto-correlation function, shown in figure 3 as well as its integral. It is readily seen that the values of the self-diffusivity resulting from the two approaches show excellent agreement and are within the margins of statistical error of the values reported by Sierou \& Brady (2004): $D_{y y}^{s}=0.0460 \pm 0.0050, D_{z z}^{s}=0.0185 \pm 0.0020$. Further, we repeated the calculations for a wide range of particle volume fractions $\phi=0.05-0.60$ with $N=512$. As discussed in Sierou \& Brady (2004), the effect of the finite size of the simulation cell on the self-diffusivity is rather weak, $D^{s} \propto\left(1-b N^{-1}\right)$, so further corrections to the infinite system limit for larger systems would be very small.

Since the extensive study of the self-diffusivity using ASD simulations in the range $\phi=0.10-0.50$ was reported earlier by Sierou \& Brady (2004), where the self-diffusivity was found by probing the time rate of change of the mean-square displacement or via the velocity autocorrelation function, we shall only compare these earlier results to those obtained here from the dynamic structure factor (DSF) approach. Also, we were able to probe the self-diffusivity beyond the order-disorder transition point in the shear-induced ordered phase at $\phi=0.55$ and in the shear-melted suspension at $\phi=0.60$. Figures $4(a)$ and $4(b)$ depict the evolution of $-k^{-2} \ln F_{s}(\boldsymbol{k}, t)$ in the $y$ and $z$-directions, respectively, as a function of time for different volume fractions. 

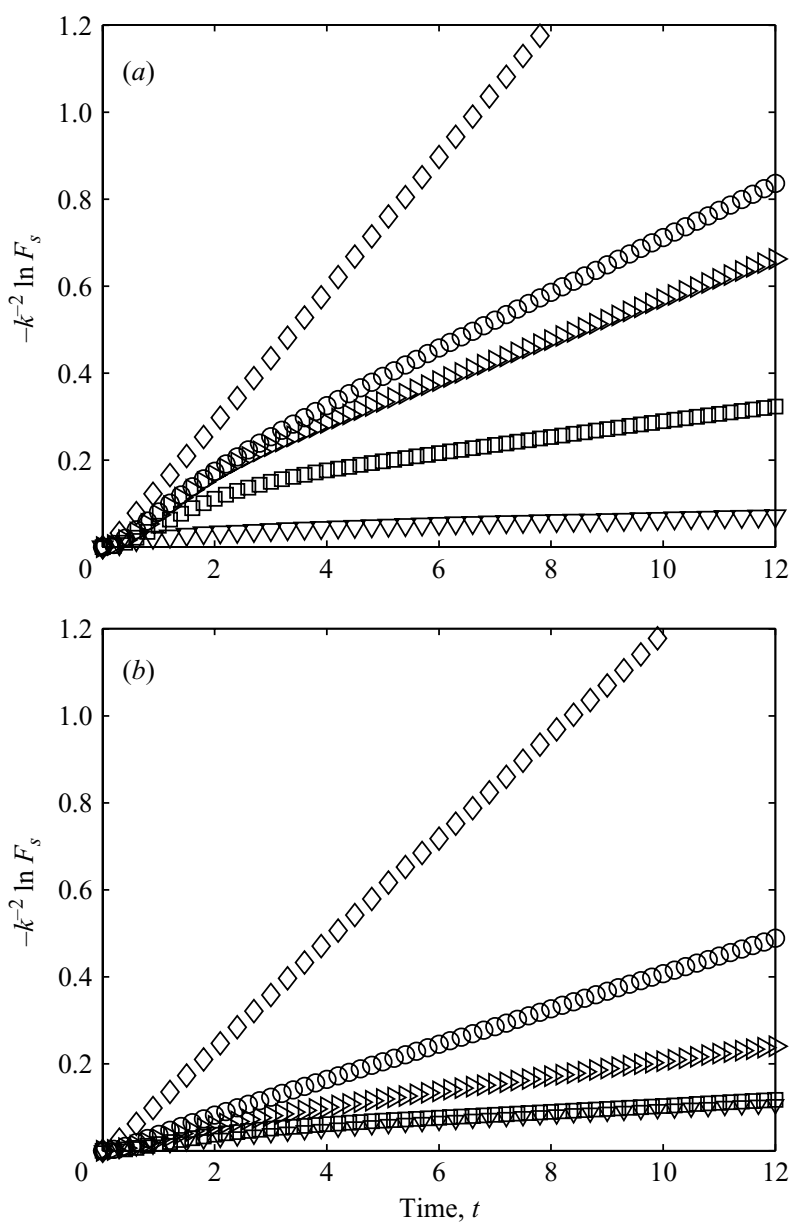

FIGURE 4. Evolution of the self-dynamic structure factor, $-k^{-2} \ln F_{s}(\boldsymbol{k}, t)$, vs. time, for $N=512$ particles and different particle volume fractions: $\phi=0.25(\square), \phi=0.35(\triangleright), \phi=0.45(\bigcirc)$, $\phi=0.55(\nabla)$ and $\phi=0.60(\diamond) .(a) \boldsymbol{k}=2 \pi / H \boldsymbol{e}_{y} ;(b) \boldsymbol{k}=2 \pi / H \boldsymbol{e}_{z}$.

It is evident from figure 4 that after a short transient the linear diffusive regime sets in. Also, the self-diffusivities in both transverse directions increase monotonically with $\phi$ up to $\phi \sim 0.40$. At $\phi=0.55$ ( $\nabla$ symbols in figure 4 ), which corresponds to the shear-induced ordered phase (Sierou \& Brady 2002), an abrupt order-of-magnitude drop in the self-diffusivity is evident in both transverse directions. The analogous observation of a rapid drop in transverse self-diffusivity related to the shear-induced ordering transition in colloidal suspension of charge-stabilized 'soft' spheres near the melting point was made in the Brownian dynamics (BD) simulations of Xue \& Grest (1990). The structure of the ordered phase is also quite similar to what was found in sheared suspensions in BD simulations (Xue \& Grest 1990; Foss \& Brady 1999) and in experiments (e.g. Ackerson \& Pusey 1988; Chen et al. 1992): there is a string ordering along the flow direction $x$, while the strings are packed into an hexagonal lattice in the transverse $(y, z)$-plane. Also, there have been a number of computational studies using non-equilibrium molecular dynamics, NEMD, (e.g. Erpenbeck 1984) that show similar string formation in molecular fluids. To observe the effect of the flow in the NEMD simulations the shear rate must be very large 


\begin{tabular}{cllcc}
\hline$\phi$ & $D_{y y}^{s}$ & \multicolumn{1}{c}{$D_{z z}^{s}$} & $D_{y y S B}^{s}$ & $D_{z z S B}^{s}$ \\
0.05 & 0.00058 & 0.00033 & & \\
0.10 & 0.0014 & 0.0010 & $0.0017 \pm 0.0003$ & $0.0011 \pm 0.0002$ \\
0.15 & 0.0040 & 0.0024 & $0.0045 \pm 0.0006$ & $0.0024 \pm 0.0004$ \\
0.20 & 0.0084 & 0.0040 & $0.0084 \pm 0.0010$ & $0.0040 \pm 0.0006$ \\
0.25 & 0.0175 & 0.0069 & $0.0171 \pm 0.0020$ & $0.0070 \pm 0.0007$ \\
0.30 & 0.0312 & 0.0115 & $0.0310 \pm 0.0040$ & $0.0117 \pm 0.0010$ \\
0.35 & 0.0467 & 0.0173 & $0.0460 \pm 0.0050$ & $0.0185 \pm 0.0020$ \\
0.40 & 0.0633 & 0.0285 & $0.0620 \pm 0.0060$ & $0.0290 \pm 0.0030$ \\
0.45 & 0.0624 & 0.0404 & $0.0583 \pm 0.0070$ & $0.0450 \pm 0.0040$ \\
0.50 & 0.0572 & 0.0502 & $0.0580 \pm 0.0070$ & $0.0520 \pm 0.0050$ \\
0.55 & 0.0027 & 0.0051 & & \\
0.60 & 0.146 & 0.125 & &
\end{tabular}

TABLE 1. Values of the self-diffusivites $D_{y y}^{s}$ and $D_{z z}^{s}$ evaluated for different volume fractions for $N=512$ using the dynamic structure factor approach. $D_{y y S B}^{s}$ and $D_{z z S B}^{s}$ correspond to the equivalent values reported in Sierou \& Brady (2004) evaluated via the time rate of change of the particle mean-square displacements.

$\left(\sim 10^{12} \mathrm{~s}^{-1}\right)$, and one needs to be concerned about the proper energy removal from the system. In fact, Evans \& Morriss (1986) found that the shear-induced alignment into strings in these studies was an artifact of the form of the temperature thermostat. They also suggest that one should not expect to find string-ordering transition in dense atomic fluids.

Although a detailed study of the suspension microstructure under shear is available in Sierou \& Brady (2002), the shear-induced ordering transition can be easily distinguished from the plots of a projection of the static structure factor $S(\boldsymbol{k})$ onto the velocity-gradient and vorticity axes. As shown in figure 5 the height of the first peak in $S\left(k_{y}\right)$ increases dramatically as particle concentration increases from $\phi=0.45$ to $\phi=0.55$, confirming that layering has occurred, while the height of the second peak of $S\left(k_{z}\right)$ exceeds the first, indicating ordering of strings into a lattice in the $(y, z)$-plane (Xue \& Grest 1990).

Increasing further the particle volume fraction up to $\phi=0.60$ while keeping the shear rate unchanged, results in shear-induced melting of the ordered phase (see Sierou \& Brady 2002) and an abrupt order-of-magnitude increase of the self-diffusivity $(\diamond$ symbols in figure 4$)$. Table 1 shows the values of the self-diffusivities evaluated for a wide range of particle volume fractions with $N=512$ via the DSF approach and the analogous results from Sierou \& Brady (2004) where the time rate of change of the particles' mean-square displacements was probed. The agreement between these results is very good and the discrepancy is always within the statistical error (not shown). In the figure 6 the dependence of $D_{y y}^{s}$ and $D_{z z}^{s}$ on the volume fraction from table 1 is shown on a $\log -\log$ plot. Below $\phi=0.10$ the self-diffusivity grows roughly as $\phi^{2}$, while beyond $\phi=0.20$ it grows approximately as $\phi^{3}$.

The presence of a plateau in $D_{y y}^{s}$ staring at $\phi \sim 0.4$ compared to monotonically increasing $D_{z z}^{s}$ up to $\phi \simeq 0.5$ probably reflects the structural changes in the suspension as the particle concentration increases. As mentioned before, the continuous increase in the first peak of $S\left(k_{y}\right)$, as shown in figures 5, suggests that some layering occurs prior to the string-ordered transition. We suggest that the anisotropy caused by layering may hinder the self-diffusivity in the velocity-gradient direction. 

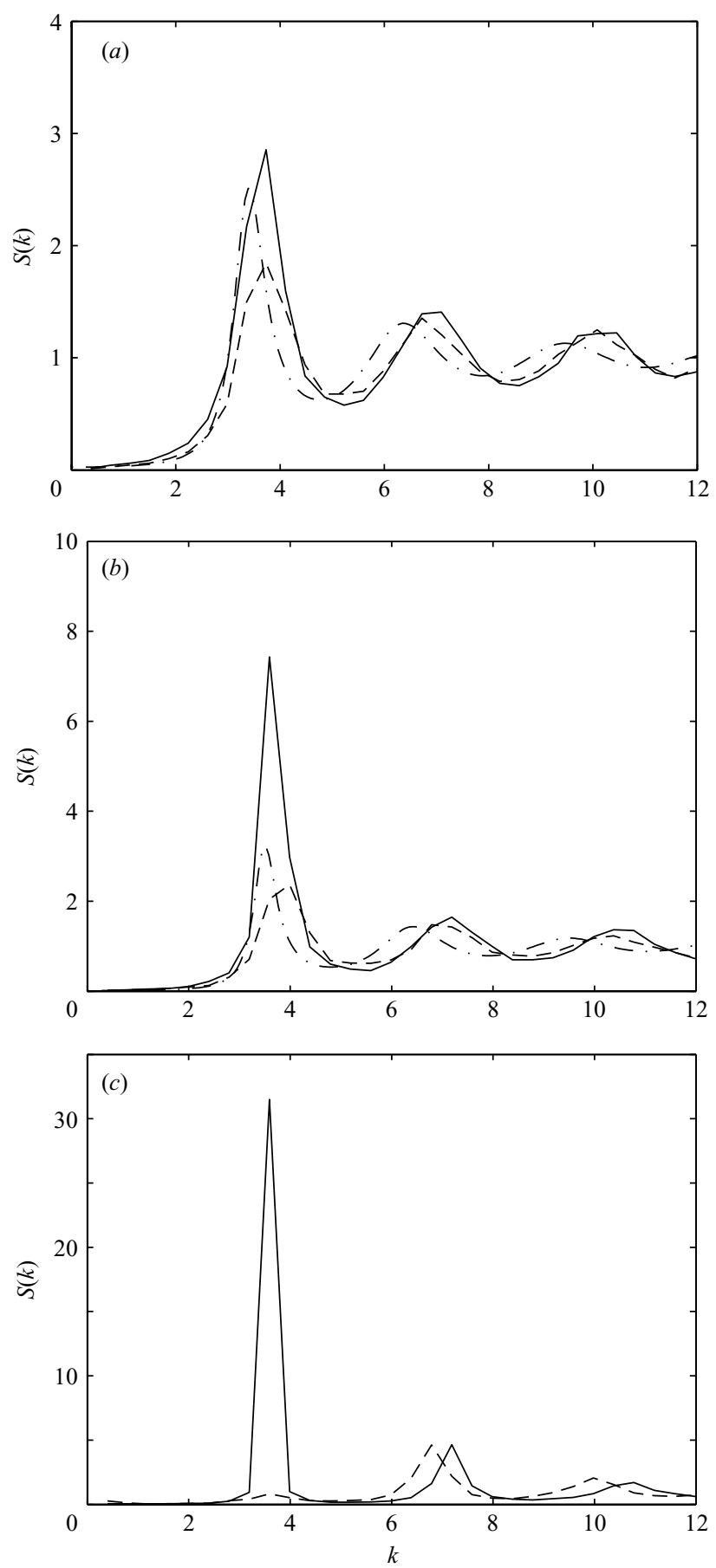

FIGURE 5. Projection of the static structure factor $S(\boldsymbol{k})$ onto velocity-gradient $(-)$ and vorticity ( ---$)$ axes, respectively; - - - Percus-Yevic closure for $S^{e q}(k)$. (a) $\phi=0.45$; (b) $\phi=0.50 ;(c) \phi=0.55$. 


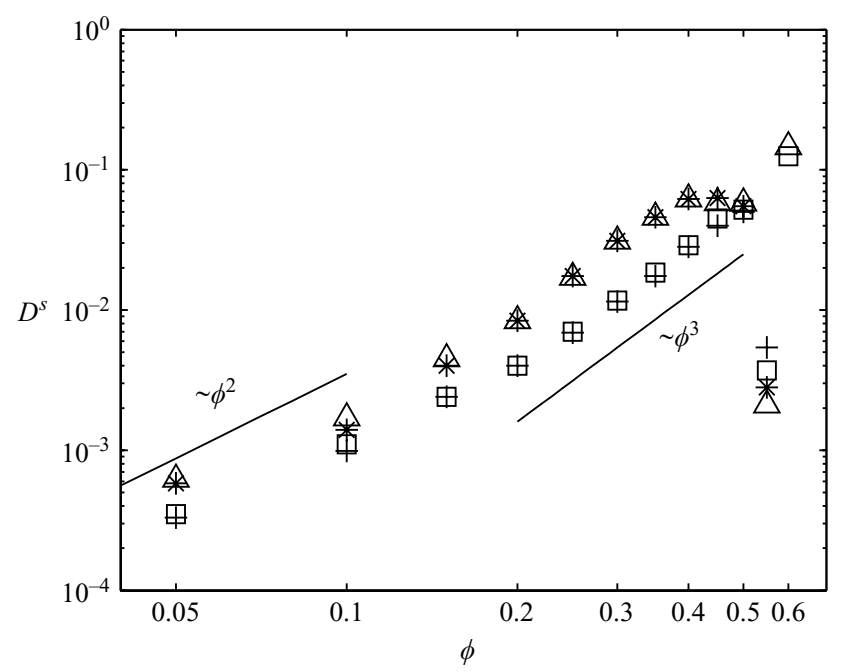

Figure 6. The self-diffusivities, $D_{y y}^{s}(*), D_{z z}^{s}(+)$, calculated from the decay of $F_{s}$ for $N=512$ compared with the equivalent results found from the mean-square displacements, $D_{y y}^{s}(\triangle)$, $D_{z z}^{s}(\square)$ as a function of volume fraction.

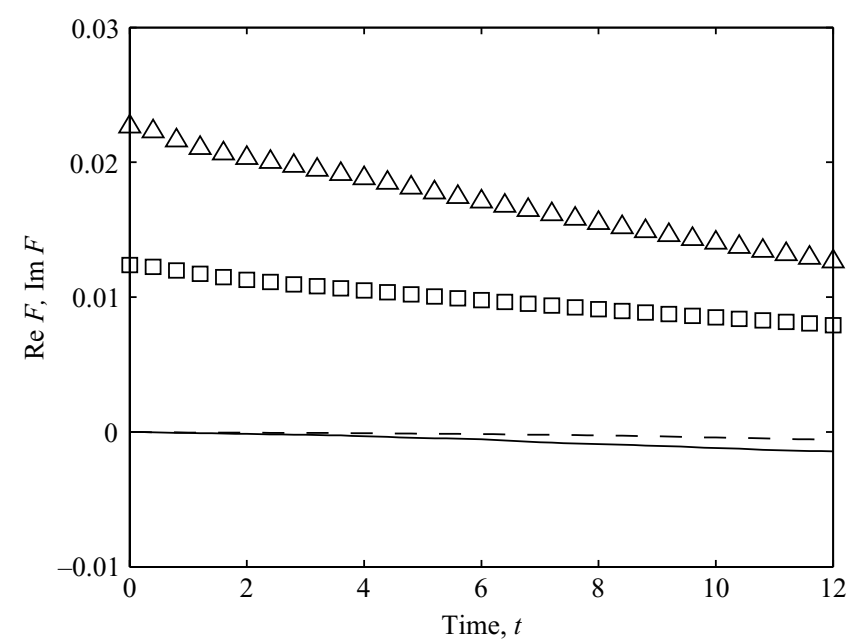

FIGURE 7. Decay of the averaged total dynamic structure factor, $F(\boldsymbol{k}, t)$, for a system $\phi=0.35$, $N=512$ and $k=2 \pi / H: \operatorname{Re} F(\boldsymbol{k}, t)$ with $\boldsymbol{k}=k \boldsymbol{e}_{y}(\triangle)$ and $\boldsymbol{k}=k \boldsymbol{e}_{z}(\square) ; \operatorname{Im} F(\boldsymbol{k}, t)$ with $\boldsymbol{k}=k \boldsymbol{e}_{y}$ $(-)$ and $\boldsymbol{k}=k \boldsymbol{e}_{z}(---)$.

Finally, to rule out the possibility of the effect of the system size on shear-induced ordering, we repeated simulations above $\phi=0.5$ for larger systems with up to $N=2048$ particles; the same results were produced as with $N=512$.

\subsection{Calculation of the gradient diffusion coefficients}

In this study the collective diffusivity in directions transverse to the direction of flow is determined via the time evolution of the dynamic structure factor, $F(\boldsymbol{k}, t)$. First, we will present in detail the results of a test case at a volume fraction $\phi=0.35$. In figure 7 we show the time evolution of $F(\boldsymbol{k}, t)$ in the $y$-and $z$-directions for $\phi=0.35$ and $N=512$. As for the self-diffusivity, the values of $F$ are averaged over all 


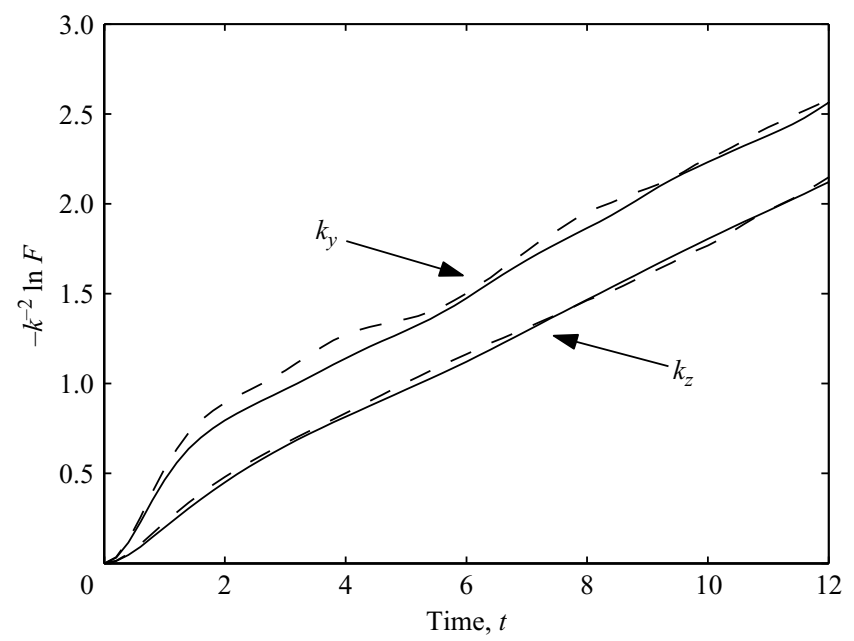

FIGURE 8 . Time evolution of the averaged total dynamic structure factor, $F(\boldsymbol{k}, t)$, probed at $k=2 \pi / H$ for $\phi=0.35, N=64$ for short and long strains. each; ---, 32 short runs of $\times 100$ strains each.

available (overlapping) time intervals in one long run (typically up to 400-800 time strains) and over several independent runs (typically 8) to further improve accuracy. As expected, the imaginary part of $F$ is very close to zero, while the real part follows the exponential decay according to (2.15). It can also be shown that time averaging over a single long run and ensemble averaging over a number of independent shorter runs are statistically equivalent. In figure 8 the comparison between these two schemes of averaging is presented for $\phi=0.35$ and $N=64$. The solid and the dashed lines correspond to the evolution of $F$ averaged over two long runs of 1600 time strains each and over 32 short runs of 100 strains each, respectively. While there is an excellent agreement between two sets of curves, averaging over a long run seems to be preferable since in this case a larger number of overlapping time intervals are available. The calculations are repeated with a varying number of particles in the simulation box, from $N=64$ to $N=2048 \dagger$ and then the collective diffusion coefficients $D_{y y}^{c}$ and $D_{z z}^{c}$ are determined as the slopes of the curves of $-k^{2} \ln F$ plotted vs. time as in figure 9. The shape of the curves in figure 9 is similar to the those in figure 4: the linear diffusive regime sets in after a short transient of $\sim 2$ strains.

The lowest $k$ accessible with current ASD simulations (e.g. $k_{c} \simeq 0.22$ for $\phi=0.35$ and $N=2048$ ) is not small enough to accurately determine the gradient diffusivity from the leading term of the asymptotic expansion of $-\dot{F} / k^{2} F$ given by (2.20), and higher-order terms in the expansion (2.18) need to be taken into account. Although (2.20) is expected to be asymptotically valid in the vicinity of $k=0$, this sets a severe requirement on the number of particles needed in the simulation box since $k_{c}=\left(6 \pi^{2} \phi / N\right)^{1 / 3}$ and, for example, to probe the time correlation of the density

$\dagger$ For large systems with $N=1024$ and $N=2048$ we use an approximate ASD algorithm with zero far-field force, $\boldsymbol{F}_{f f}=0$ (for ASD details see Sierou \& Brady 2001). It appears that for simple shear flow with neutrally buoyant particles the magnitude of $\boldsymbol{F}_{f f}$ is always small and, for instance, the values of the self-diffivities, $D_{y y, z z}^{s}$, determined using a zero-far-field-force approximation are within the $5 \%$ error margin of those found using complete ASD scheme. Use of this approximation allows accurate $O(N)$ computations for large systems, but is restricted to the flows with force-free particles. 

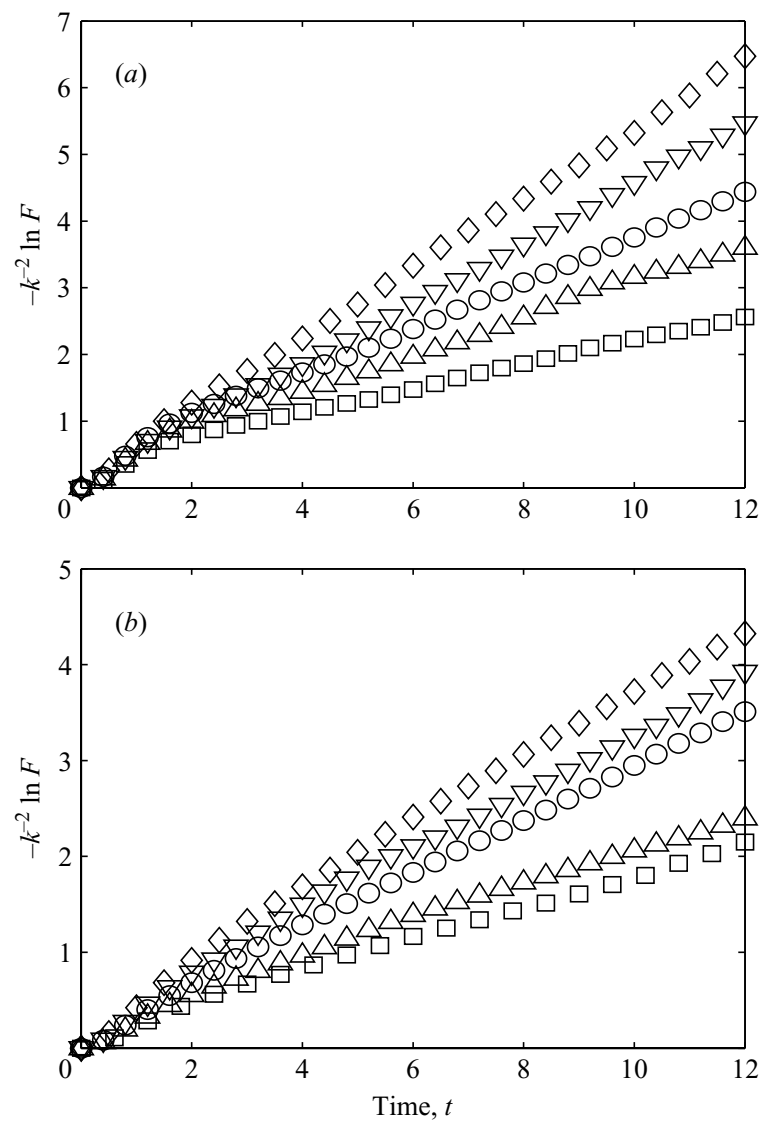

Figure 9. Computed values of $-k^{-2} \ln F$ plotted vs. time $t$ for volume fraction $\phi=0.35$ and different number of particles: $N=64(\square), N=128(\triangle), N=256(\bigcirc), N=512(\triangleright)$ and $N=1024(\diamond)$. The collective diffusivity is determined as a slope of the corresponding curve. (a) $\boldsymbol{k}=2 \pi / H \boldsymbol{e}_{y} ;(b) \boldsymbol{k}=2 \pi / H \boldsymbol{e}_{z}$.

fluctuations with $k \lesssim 0.1$ for $\phi=0.35$ one must be able to simulate systems with $N \sim 10^{4}$ particles in a cubic cell, which severely limits the number of simulations that can be performed. Note, that this differs from the self-diffusivity where the leading term of the analogous low- $k$ expansion of $-\dot{F}_{s} / k^{2} F_{s}$ provides very good results up to moderate $k \sim O(1)$.

It appears, however, that values of the collective diffusivity determined using a two-term expansion in (2.18) are within the statistical accuracy of those found from direct measurements of the decay of $F$. In figure 10 the two-term expansion of $\dot{F} / k^{2} F$ in (2.18) is plotted as a function of time for $\boldsymbol{k}=k \boldsymbol{e}_{y}$. As expected from the asymptotic expression (2.20), the form of the curves resembles the integral of the velocity autocorrelation shown in figure 3 and the plateau value in the long-time limit equals the collective diffusion coefficient. An analogous figure (not presented) is obtained for the collective diffusivity in the vorticity direction.

Finally, the values of the collective diffusion coefficients in both transverse directions found from the decay of the DSF are plotted as a function of the number of particles in a simulation cell, $N$, in figure 11 . It is readily seen that the data are fairly well approximated by the fit $D^{\nabla}\left(1-b N^{-1 / 3}\right)$, where $D^{\nabla}$ is a gradient diffusivity 


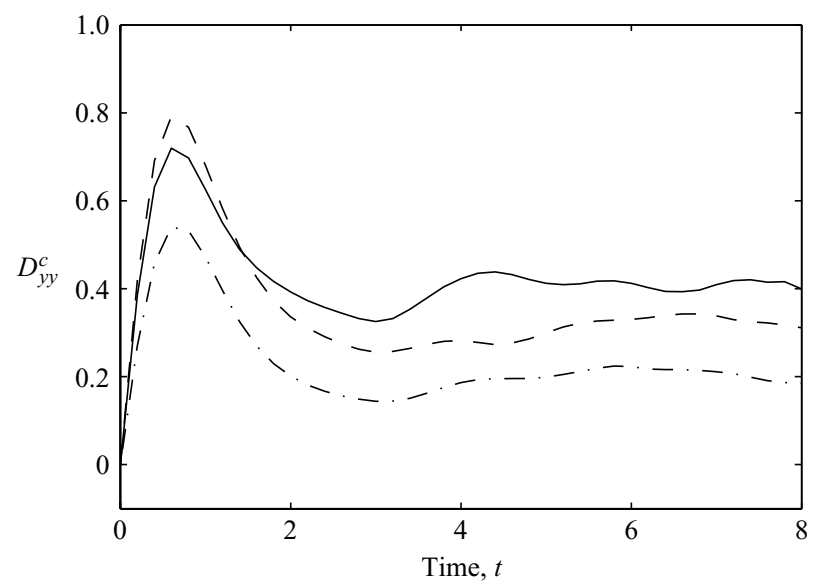

FIGURE 10. Collective diffusivity in the velocity-gradient direction, $D_{y y}^{c}$, for the volume fraction $\phi=0.35$ calculated according to $(2.18)$ for different number of particles: $N=64(-\cdot-)$, $N=256(---)$ and $N=512\left(-\frac{-}{-}\right.$.

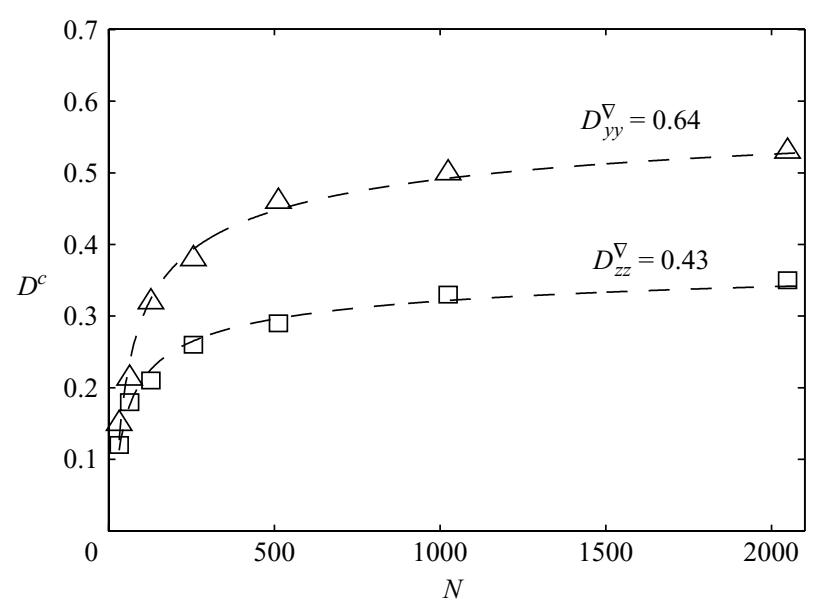

FIGURE 11. Collective diffusivity for volume fraction $\phi=0.35$ plotted as a function of the number of particles in a simulation box. $D_{y y}^{c}(\triangle), D_{z z}^{c}(\square)$. Dashed lines are the extrapolation curves corresponding to $D^{\nabla}\left(1-b N^{-1 / 3}\right)$. The values of the extrapolated gradient diffusivities are also shown.

corresponding to $k=0$. The values of the extrapolated gradient diffusivities are also shown in the figure.

It is evident from figure 11 that the dependence on the system size is different $\left(\sim N^{-1 / 3}\right)$ from that for the self-diffusivity $\left(\sim N^{-1}\right)$. Let us recall that unlike the selfdiffusivity where $\boldsymbol{D}^{s}$ is independent of the wavelength of the probed fluctuation of the tagged particle number density, for collective diffusion, apart from the finite-box-size effects associated with the periodicity of the boundaries, $\boldsymbol{D}^{c}$ itself is $k$-dependent. We use $\boldsymbol{D}^{c}$ here to denote the value of diffusivity determined from the DSF approach before the small- $k$ limit is taken. This dependence can be separated from the finitebox-size effects if, for instance, the time evolution of $F$ is probed at the same $k$ upon varying the number of particles in the simulation box. Since $k$ can take on discrete 


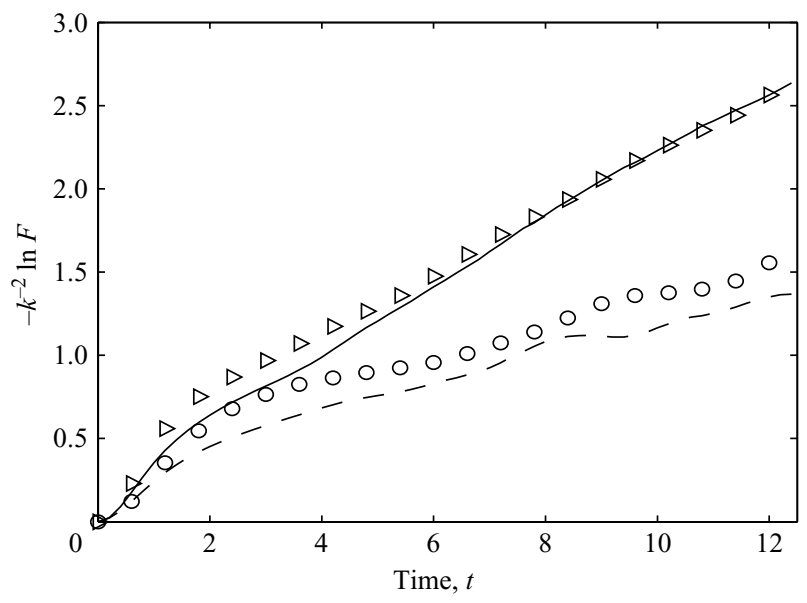

FiguRE 12. Time evolution of the total dynamic structure factor, $F(\boldsymbol{k}, t)$, for $\phi=0.35$ probed at the same wavenumber, $\boldsymbol{k}=k \boldsymbol{e}_{y}$, upon varying the number of particles. $N=512: k=4 \pi$ / $H=0.687(\longrightarrow), k=8 \pi / H=1.37(---) ; N=64: k=2 \pi / H=0.687(\triangleright), k=4 \pi / H=1.37(\bigcirc)$.

$\begin{array}{ccc}\phi & D_{y y}^{\nabla} & D_{z z}^{\nabla} \\ 0.20 & 0.068 \pm 0.008 & 0.032 \pm 0.004 \\ 0.25 & 0.147 \pm 0.006 & 0.089 \pm 0.004 \\ 0.30 & 0.32 \pm 0.02 & 0.190 \pm 0.015 \\ 0.35 & 0.64 \pm 0.05 & 0.43 \pm 0.09 \\ 0.40 & 0.83 \pm 0.10 & 0.76 \pm 0.15 \\ 0.45 & 1.04 \pm 0.14 & 1.37 \pm 0.21 \\ 0.50 & \sim 1.6 & \sim 2.3\end{array}$

TABLE 2. Summary of the values of the extrapolated gradient diffusivities $D_{y y}^{\nabla}$ and $D_{z z}^{\nabla}$ evaluated for different volume fractions $\phi$.

values $2 \pi m / H$, where $m=1,2,3, \ldots$, and since $k \propto(\phi / N)^{1 / 3}$, to be able to probe $F$ at the same wavenumber at different $N$, the number of particles in a simulation should vary by multiples of $2^{3}$. Thus, the time evolution of $F$ is probed at two distinct values of the wavenumber, $k=0.687$ and $k=1.37$, for the volume fraction $\phi=0.35$ and two different box sizes corresponding to $N=512$ and $N=64$. As one can see from figure 12 the finite-box-size effects are negligible, and the variation of $D^{c}$ (already with $N \sim 10^{2}$ ) is entirely due to the chosen wavelength of the density fluctuation $k$.

The calculations of $F$ are performed for a range of volume fractions (up to $\phi=0.50$ ) and wavenumbers (down to $k=0.2$ ) and the values of the collective diffusivities $D_{y y}^{c}$ and $D_{z z}^{c}$ are plotted as a function of wavenumber $k$ in figures $13(a)$ and $13(b)$, respectively. It is evident that the linear regression $D^{\nabla}(1-b k)$ (which is equivalent to $N^{-1 / 3}$ extrapolation) is not accurate for the entire range of the volume fractions: above $\phi=0.40$ the curves $D^{c}$ vs. $k$ deform and level out as $k \rightarrow 0$ and this is accounted for by using a Padé approximant of the form $\left(a_{0}+a_{1} k+\cdots+a_{i} k^{i-1}\right) /\left(b_{0}+\right.$ $\left.b_{1} k+\cdots+b_{i+1} k^{i}\right)$. An analogous tendency is observed for the dependence $D_{z z}^{c}$ vs. $k$ in figure $13(b)$ and the same extrapolation procedure is applied in this case as well. The resulting values of the extrapolated gradient diffusivity are provided in table 2 . 

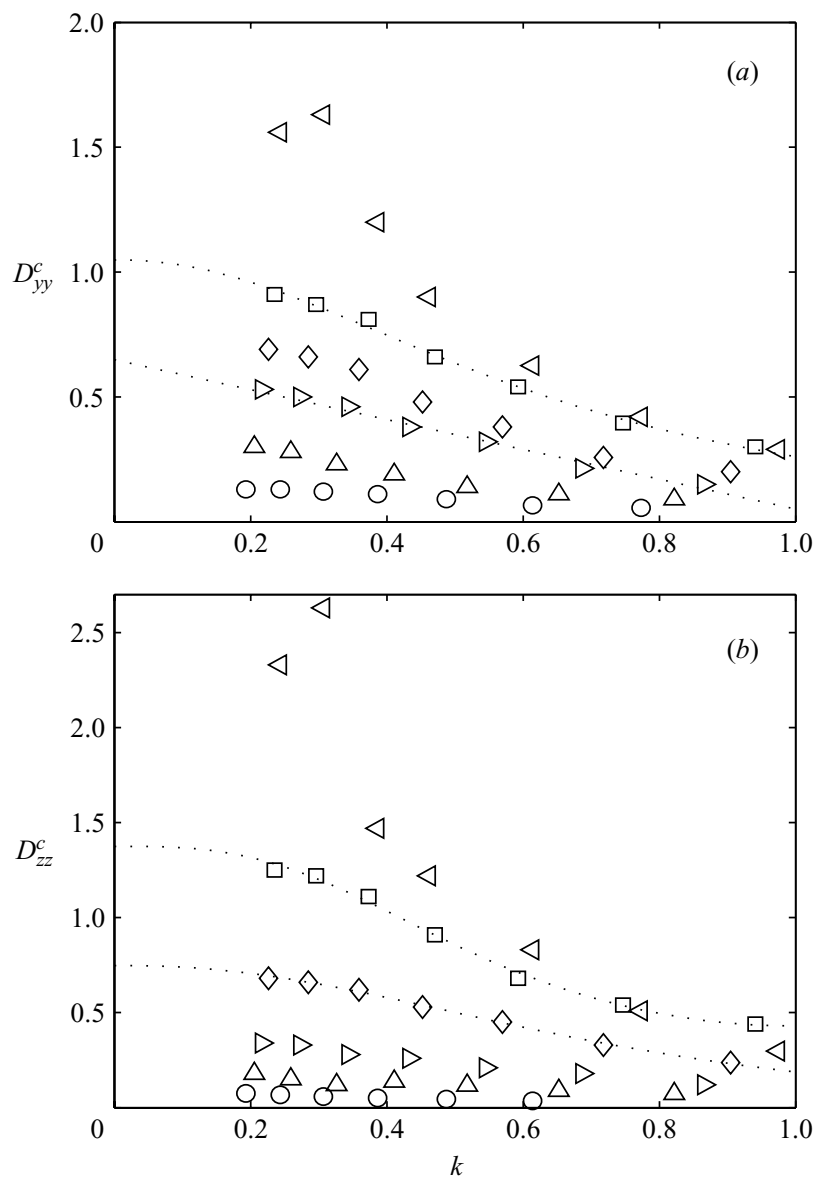

FIGURE 13. Transverse collective diffusivity as a function of a wavenumber, $k$, for different volume fractions: $\phi=0.25(\bigcirc), \phi=0.30(\nabla), \phi=0.35(\triangleright), \phi=0.40(\diamond), \phi=0.45(\square)$ and $\phi=0.50(\triangleleft)$. Dotted lines show the extrapolation to the $k=0$ limit. (a) velocity-gradient direction; $(b)$ vorticity direction.

In figure 14 values of the gradient diffusivities from table 2 are depicted vs. $\phi$ on a log-log plot and compared to the available experimental results (Leighton \& Acrivos 1987; B. K. Chapman \& D. T. Leighton 1991, personal communication; Phillips et al. 1992) and earlier numerical calculations by Marchioro \& Acrivos (2001). The agreement between the experimental measurements and the results of the present study is quite good over the entire range of volume fractions. It should be mentioned, however, that none of the experimental gradient diffusivities presented in figure 14 are from direct measurements of the diffusivities. The experiments involve either macroscopic concentration gradients (Leighton \& Acrivos 1987, Chapman \& Leighton) or spatially varying shear gradients (Phillips et al. 1992), and the diffusivities are extracted from fitting particle migration data to the solution of model equations.

Note, that the values of the gradient diffusivity are typically more than an order-ofmagnitude larger than those of the self-diffusivity. This can be readily explained using the asymptotic expression for the collective diffusivity in the low- $k$ limit (2.20). Indeed, the so-called hydrodynamic factor $\mathscr{H}$ defined in (2.20) can be decomposed into a sum of the velocity auto-correlation term ( $\alpha=\beta$ only) and the velocity cross-correlation 


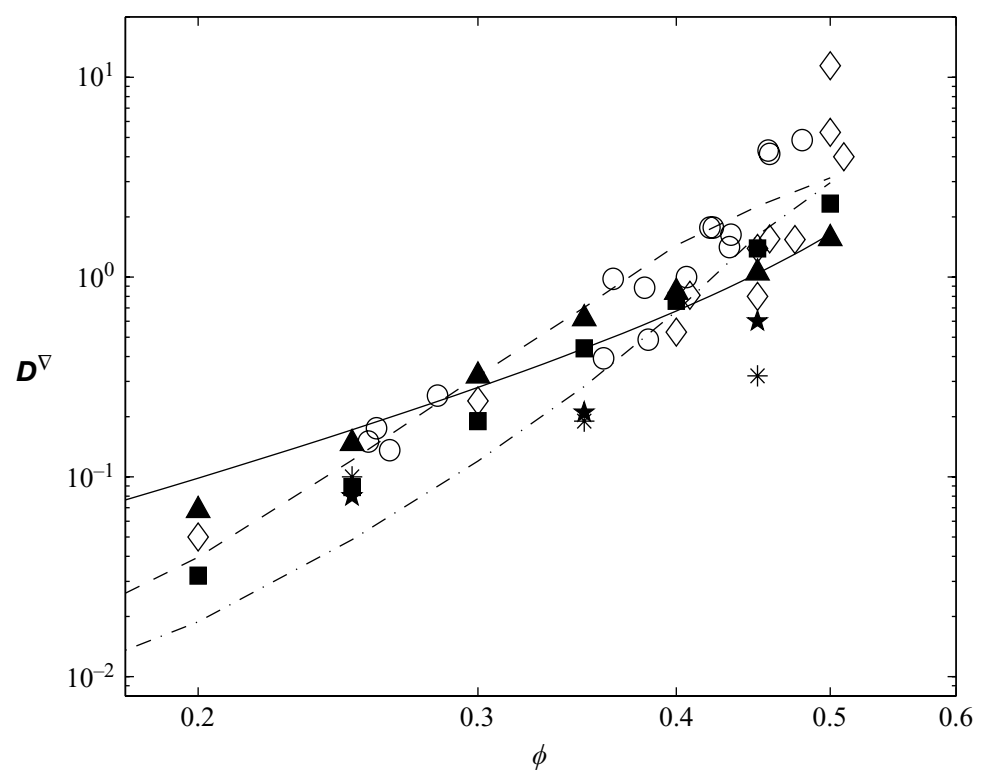

Figure 14. The dependence of gradient diffusivity, $\boldsymbol{D}^{\nabla}$ on the particle volume fraction $\phi$. Experimental results: Leighton \& Acrivos (1987) (O), B. K. Chapman \& D. T. Leighton (1991, personal communication) $(\diamond)$, Phillips et al. (1992) (-); ASD results of the present study: $D_{y y}^{\nabla}(\mathbf{\Lambda}), D_{z z}^{\nabla}(\mathbf{\square})$; SD results of Marchioro \& Acrivos $(2001): D_{y y}^{\nabla}(*), D_{z z}^{\nabla}(\star)$; approximate model: $D_{y y}^{s} z S^{e q}(0)(---)$ and $D_{z z}^{s} / S^{e q}(0)(-\cdot-)$.

term $(\alpha \neq \beta$ only $)$ as

$$
\mathscr{H}=\boldsymbol{D}^{s}+\frac{1}{N}\left\langle\sum_{\alpha \neq \beta} \mathscr{M}_{\alpha \beta} \exp \left[\mathrm{i} \boldsymbol{k} \cdot\left(\boldsymbol{x}_{\alpha}-\boldsymbol{x}_{\beta}\right)\right]\right\rangle,
$$

and therefore

$$
\boldsymbol{D}^{c}=\frac{\mathscr{H}(\boldsymbol{k}, t)}{S(\boldsymbol{k})}=S(\boldsymbol{k})^{-1}\left(\boldsymbol{D}^{s}+\text { cross-terms }\right) .
$$

Thus, if the contribution from the cross-terms is neglected, in the limit $t \rightarrow \infty, k \rightarrow 0$ we can approximate the gradient diffusivity as $\boldsymbol{D}^{\nabla} \sim \boldsymbol{D}^{s} / S(0)$, where $S(0)$ is the nonequilibrium value of the static structure factor at zero wavevector, which can be determined numerically from (2.19) extrapolating to $k=0$. Our calculations of the static structure factor of strongly sheared suspensions of hard spheres (see figure 17) suggest that the long-wavelength fluctuations in the particle number density are unaffected by shear, i.e. $S(\boldsymbol{k}) \sim S^{e q}(k)$ as $k \rightarrow 0$ which is in accord with experimental results (e.g. Wagner \& Russel 1990). Although we cannot probe $S(k)$ in the near vicinity of $k=0$, we shall use the equilibrium value of $S^{e q}(0)$ for hard spheres that can be estimated from the Carnahan-Starling approximation (Pusey 1991). The values of $D_{y y}^{s} /$ $S^{e q}(0)$ and $D_{z z}^{s} / S^{e q}(0)$ are plotted in figure 14 (the dashed and the dashed-dotted lines, respectively). The qualitative agreement of this approximation with the experimental results and the results of the DSF approach is fairly good, which means that the underlying physical mechanism of the shear-induced gradient diffusion is similar to that in equilibrium colloidal dispersions: the driving force is the non-equilibrium osmotic compressibility, $\partial \Pi / \partial n \propto S(0)^{-1}$, while the effect of the collective mobility is well approximated by the shear-induced self-mobility of the particles. 


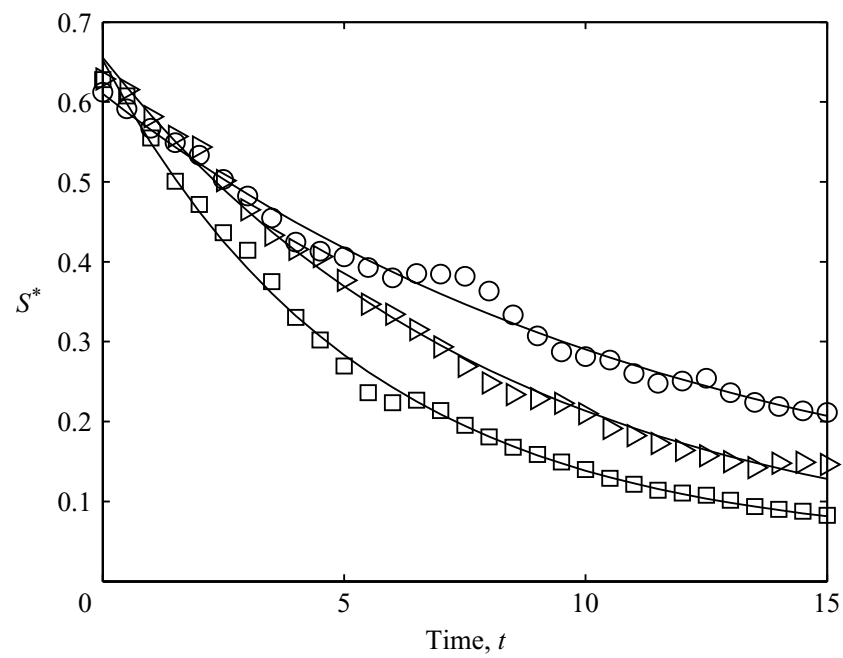

FIgURE 15. Computed values of $S^{*}(\boldsymbol{k}, t)$ for a volume fraction $\phi=0.35, k=2 \pi / H \boldsymbol{e}_{y}$ and different number of particles, $N$, and initial configurations, $N_{c}: N=64, N_{c}=132(\square) ; N=256$, $N_{c}=32(\triangleright) ; N=512, N_{c}=16(\bigcirc)$. The solid lines are the best fit according to (5.2).

\section{Alternative method of Marchioro \& Acrivos (2001) revisited}

As seen in figure 14 the agreement with the experimental measurements is very good, while there is considerable discrepancy between the results of the present study and the earlier numerical results of Marchioro \& Acrivos (2001). In their study Marchioro \& Acrivos (2001) applied a different approach to determine the gradient diffusivity from conventional SD simulations. Their approach is based upon probing the temporal relaxation of the quantity $S^{*}(t)$, defined as

$$
S^{*}(\boldsymbol{k}, t)=\frac{2}{N}\left\langle\left|\sum_{\alpha=1}^{N} \sin \boldsymbol{k} \cdot \boldsymbol{x}_{\alpha}(t)\right|^{2}\right\rangle,
$$

where $\boldsymbol{k}=2 \pi / H \boldsymbol{e}_{y, z}$. It was argued by Marchioro \& Acrivos (2001) that the time relaxation of $S^{*}$ is diffusive and follows

$$
S^{*}(\boldsymbol{k}, t)=S_{\infty}^{*}+\left(S^{*}(\boldsymbol{k}, 0)-S_{\infty}^{*}(\boldsymbol{k})\right) \exp \left[-2 \boldsymbol{k} \boldsymbol{k}: \boldsymbol{D}^{c} t\right],
$$

where $S_{\infty}^{*}(\boldsymbol{k})$ is the value of $S^{*}$ in a steadily sheared suspension as $t \rightarrow \infty$ and $S^{*}(\boldsymbol{k}, 0)$ is the initial value at $t=0$. To be able to probe the collective diffusivity from the decay of $S^{*}$ the 'gain', $S^{*}(\boldsymbol{k}, 0)-S_{\infty}^{*}(\boldsymbol{k})$, in (5.2) cannot be too small. To achieve this, it was proposed to bias initial random hard-sphere configurations with a value of $S^{*}(\boldsymbol{k}, 0)$ in the range $0.5-1.0$. As a result of the lower computational efficiency of conventional SD compared to that of ASD, the number of particles used in the simulations by Marchioro \& Acrivos (2001) did not exceed $N=64$. To address the aforementioned discrepancy we performed calculations for two volume fractions $\phi=0.35$ and $\phi=0.45$ using ASD with the number of particles ranging from $N=64$ to $N=512$, while using (5.2) to extract the diffusivity. In figure 15 the time evolution of $S^{*}(\boldsymbol{k}, t)$, starting from the initial configurations with $S^{*}(0) \in(0.5,1)$ in the $y$-direction, is shown for $k_{c}=2 \pi / H \boldsymbol{e}_{y}$ and different numbers of particles. The solid lines are best fits according to (5.2). The analogous computation was performed in the vorticity direction with $k_{c}=2 \pi / H \boldsymbol{e}_{z}$ and $S^{*}(\boldsymbol{k}, 0) \in(0.5,1)$. The resulting values of the collective 

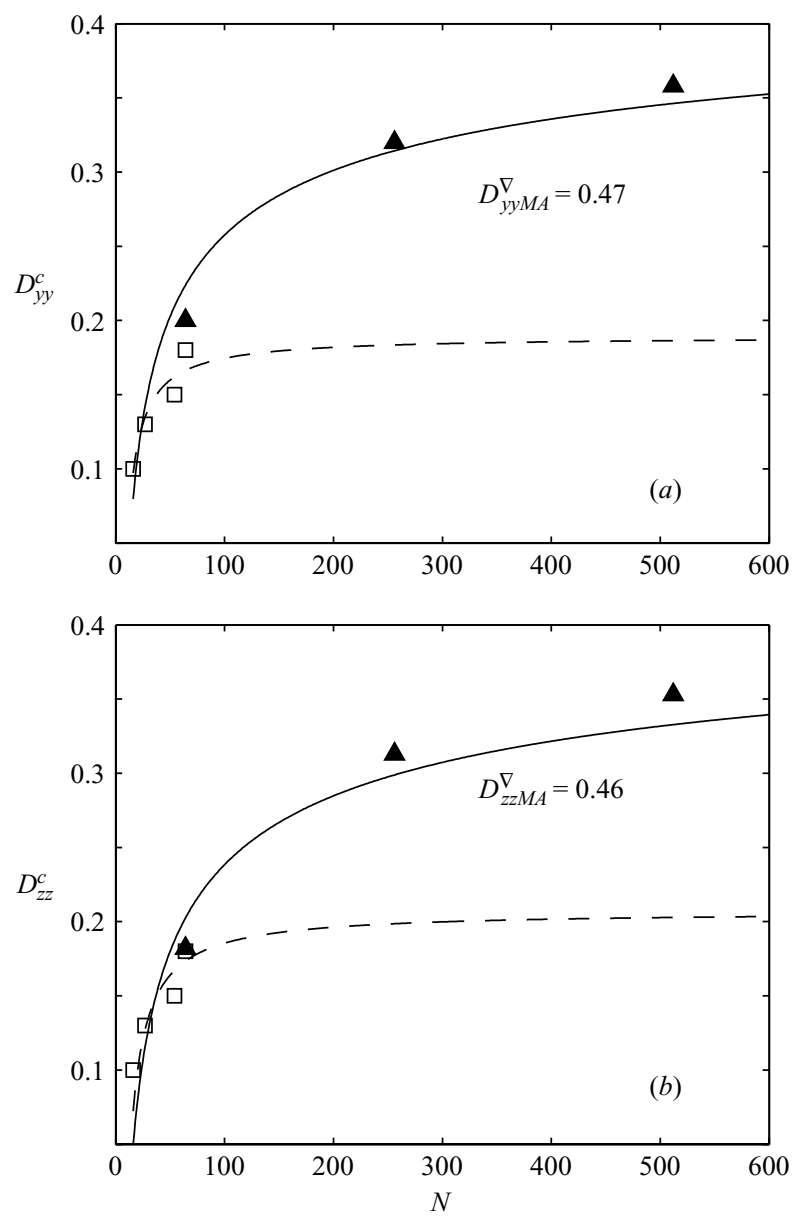

FIGURE 16. Values of collective diffusivities for a volume fraction $\phi=0.35$ plotted as a function of the number of particles: SD simulations of Marchioro \& Acrivos 2001 ( $\square$ ), ASD simulations of the present study $(\boldsymbol{\Lambda})$. The solid lines are the best fit according to $N^{-1 / 3}$, the dashed lines are the extrapolating curves $N^{-1}$ used by Marchioro \& Acrivos (2001). (a) $D_{y y M A}^{c}$; (b) $D_{z z M A}^{c}$.

diffusion coefficients, $D_{y y M A}^{c}$ and $D_{z z M A}^{c}$ (the subindex MA is used to distinguish these results from the those of the DSF approach) are depicted in figures $16(a)$ and $16(b)$, respectively, and compared with the results of Marchioro \& Acrivos (2001).

It is seen from these figures that there is a good match between the present ASD results and the SD results by Marchioro \& Acrivos (2001) at $N=64$. Since simulations in Marchioro \& Acrivos (2001) were limited to rather small systems with $N=64$ particles at most, the self-diffusivity extrapolation $N^{-1}$ (Sierou $\&$ Brady 2004) was used to estimate the gradient diffusivity in the limit of infinite suspension (dashed line in figures $16 a$ and $b$ ). It appears that the $k$-dependence is the same as for the DSF approach and the proper extrapolation curve approaches $D^{\nabla}$ as $N^{-1 / 3}$ (solid line in figure $16 a, b)$. In table 3 the values of the corrected gradient diffusivities extrapolated using the $N^{-1 / 3}$ asymptote are compared with those from Marchioro \& Acrivos (2001) and with those from the DSF approach for $\phi=0.35,0.45$. Although the corrected values of the gradient diffusivities, $D_{M A}^{\nabla c o r r}$, and the predictions of the DSF approach 


\begin{tabular}{ccccccc}
\hline$\phi$ & $D_{y y M A}^{\nabla}$ & $D_{y y M A}^{\nabla c o r r}$ & $D_{y y}^{\nabla}$ & $D_{z z M A}^{\nabla}$ & $D_{z z M A}^{\nabla c o r r}$ & $D_{z z}^{\nabla}$ \\
0.35 & $0.19 \pm 0.05$ & $0.46 \pm 0.04$ & $0.64 \pm 0.05$ & $0.21 \pm 0.02$ & $0.46 \pm 0.03$ & $0.43 \pm 0.09$ \\
0.45 & $0.32 \pm 0.08$ & $1.06 \pm 0.06$ & $1.04 \pm 0.14$ & $0.62 \pm 0.10$ & $1.36 \pm 0.16$ & $1.37 \pm 0.21$ \\
TABLE 3. Values of the gradient diffusivities evaluated using the approach of Marchioro \& \\
Acrivos (2001): $D_{M A}^{\nabla}$ correspond to the SD results obtained in their original paper by linear \\
extrapolation $N^{-1} ; D_{M A}^{\nabla \text { corr }}$ are the ASD results of the present study extrapolated by $N^{-1 / 3}$. \\
$D^{\nabla}$ correspond to the results obtained from dynamic structure factor approach.
\end{tabular}

show quite good agreement, one might expect to have very close agreement if the two approaches for determining the gradient diffusivity were equivalent. As we show below, the two approaches are not equivalent as the method of (Marchioro \& Acrivos 2001) probes relaxation of the particle number density, rather than relaxation of the particle density fluctuations probed by the DSF approach, the latter being appropriate in the weak gradient limit.

To understand this difference, we note that the quantity $S^{*}$ defined in (5.1) is related to the static structure factor $S(\boldsymbol{k})(2.19)$ and more rigorously satisfies the inequality

$$
S^{*}(\boldsymbol{k}, t) \leqslant 2 S(\boldsymbol{k}),
$$

where by $S(\boldsymbol{k})$ we mean the instantaneous value of the static structure factor at time $t$. In a steadily sheared suspension in the limit $t \rightarrow \infty$ the microstructure is different from that of the equilibrium state (see figure 1) and, moreover, in the former case the structure factor is a function of $\boldsymbol{k}$ due to the flow-induced anisotropy, while in the equilibrium case it is a function of $k=|\boldsymbol{k}|$ due to rotational symmetry. Figure 17(a) shows projections of $S(\boldsymbol{k})$ onto the three principal axes, $x, y$ and $z$, plotted as a function of wavenumber $k$ together with the equilibrium dependence $S^{e q}(k)$ calculated using the Percus-Yevic (PY) theory. The deviation $S-S^{e q}$, which is widely used in sheared colloidal dispersions to quantify the distortion of the suspension microstructure out of equilibrium, is depicted in figure $17(b)$. One can see that in addition to the distortion along the flow direction, there is a considerable perturbation along both transverse directions $y$ and $z$. Note, that in the low- $k$ limit the distortion $S-S^{e q}$ in all directions is small, while $S(\boldsymbol{k})<S^{e q}(0)$ for finite $k_{y}$ and $k_{z}$. (A qualitatively similar deformation of the microstructure was observed in experiments in steadily sheared concentrated colloidal suspensions using small-angle neutron scattering (Johnson, de Kruif \& May 1988; de Kruif et al. 1990; Wagner \& Russel 1990). As seen in figure 17(a) the peak in $S(\boldsymbol{k})$ along the flow and the vorticity directions decreases and shifts to higher $k a$ at higher shear rates (higher Péclet numbers). Also, low- $k$ measurements at $k a=0.85$ show that the ratio of the measured static structure factor for sheared dispersions to the one at equilibrium $S(\boldsymbol{k}) / S^{e q}(k)>1$ along the flow direction and $S(\boldsymbol{k}) / S^{e q}(k)<1$ along the vorticity direction (Johnson et al. 1988).) Thus, it follows from the inequality (5.3) that initial configurations with $S^{*}(\boldsymbol{k}, 0)>0.5$ would necessarily have $S(\boldsymbol{k})>0.25$ which is at least an order-of-magnitude higher than the value of $S(\boldsymbol{k})$ in a steadily sheared suspension as $t \rightarrow \infty$ (e.g. for 512 particles and $k_{c}=0.34$ we found $S(\boldsymbol{k}) \simeq 0.02$ for both $k_{y}$ and $k_{z}$, while $S^{e q}(0)=0.062$ from PY theory). In other words, although the initial configurations are picked randomly from independent hard-sphere configurations, the restriction on $S^{*}(\boldsymbol{k}, 0)$ implies that the averaged microstructure of the suspension at $t=0$ is highly perturbed compared to that at $t \rightarrow \infty$. Thus, the method proposed by Marchioro \& Acrivos (2001) concerns relaxation of the disturbed suspension microstructure, while the DSF approach applies in the steadily sheared suspension 

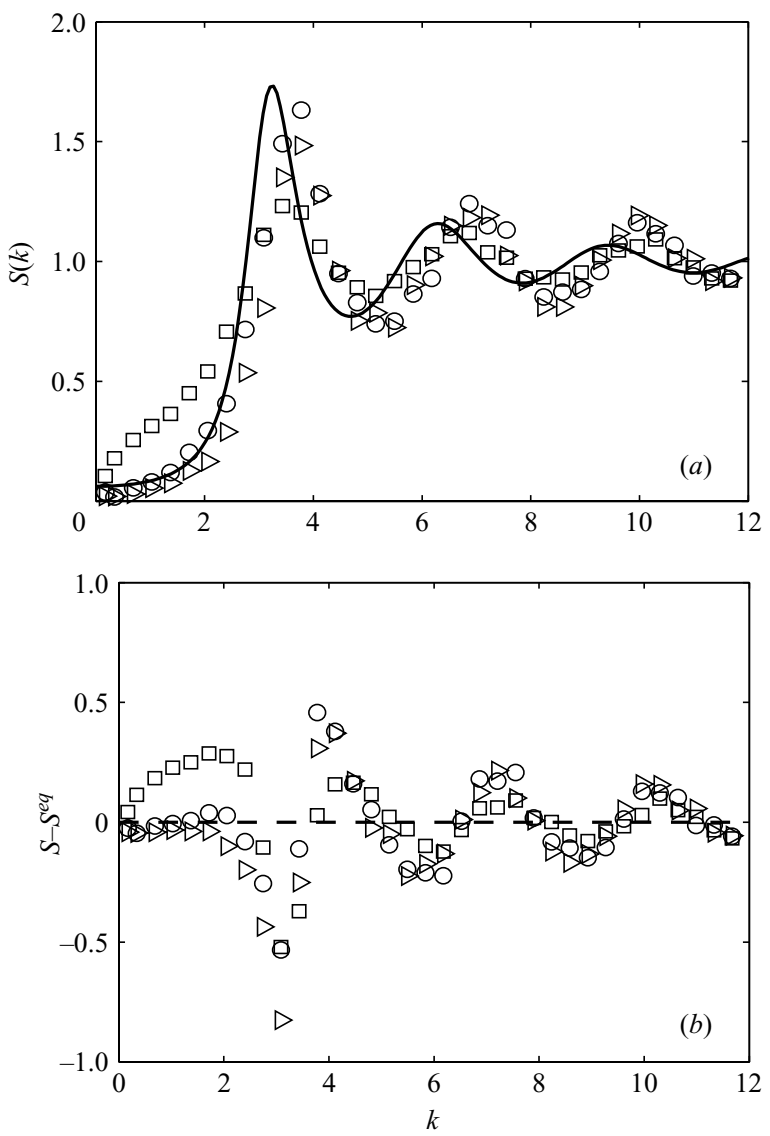

FiguRE 17. Flow-induced distortion of the suspension microstructure out of equilibrium for $\phi=0.35$. (a) Projections of the static structure factor $S(\boldsymbol{k})$ on the principal axes: in the flow direction $(\square)$, velocity-gradient $(\bigcirc)$ and vorticity $(\triangleright)$. The solid line is the Percus-Yevic closure for $S^{e q}(k)$. (b) Distortion of the static structure factor from the equilibrium state $S(k)-S^{e q}(k)$ as a function of the wavenumber $k$.

when $S(\boldsymbol{k})$ does not evolve considerably in time but only fluctuates around its mean value.

Since spatial inhomogeneity of the particle density is involved in the approach by Marchioro \& Acrivos (2001), the value of $D^{c}$ may depend on the amplitude as well as the shape of the initial distortion of the microstructure, $S^{*}(\boldsymbol{k}, 0)$. To demonstrate that the nature of the initial distortion may affect the results we performed a simulation for $\phi=0.35$ and $N=512$ particles using $N_{c}=24$ initial random hardsphere configurations with $S^{*}(\boldsymbol{k}, 0) \sim 0.45$ in both transverse directions $y$ and $z$ simultaneoulsy. $\dagger$ In this way, both coefficients $D_{y y M A}^{c}$ and $D_{z z M A}^{c}$ are evaluated from the same series of simulations. The time evolution of the ensemble-averaged $S^{*}$ is given in figure 18 for both $y$ and $z$. It appears that the symmetric distortion of the microstructure along both transverse directions results in values of collective diffusivities

$\dagger$ The generation of random hard-sphere configurations with values of $S^{*}(\boldsymbol{k}, 0) \in(0.5,1)$ along both transverse directions simultaneously is a difficult task. For $\phi=0.35$ we could not generate any random hard-sphere configurations with both $S^{*}\left(k_{y}, 0\right)$ and $S^{*}\left(k_{z}, 0\right)$ above 0.6 . This observation indicates the fact that the microstructure of such particle distributions is indeed extremely distorted. 


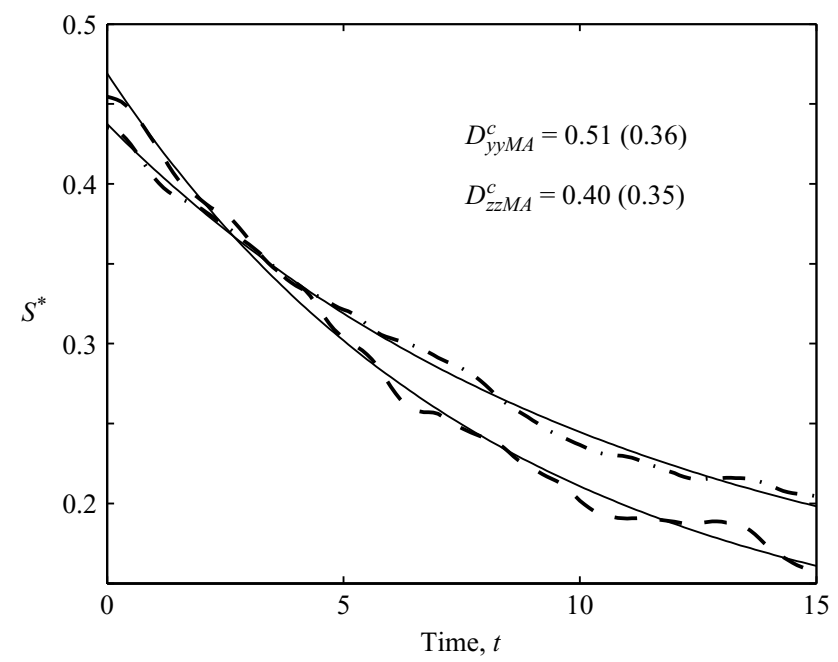

FIGURE 18. Values of $S^{*}(t)$ computed for $\phi=0.35$ and $N=512 . \boldsymbol{k}=2 \pi / H \boldsymbol{e}_{y}(---)$ and $\boldsymbol{k}=2 \pi / H \boldsymbol{e}_{z}(-\cdot-) .24$ initial random configurations with values of $S^{*}(\boldsymbol{k}, 0)$ approximately 0.45 in both directions $y$ and $z$ simultaneously are used for simulation. The resulting values of the collective diffusivities are also shown together with the equivalent results obtained with $S^{*}(\boldsymbol{k}, 0) \sim 0.5$ along only one direction $y$ or $z$ (in parenthesis).

that are quite different from those obtained using the method described by Marchioro \& Acrivos (2001) where the microstructure is initially distorted along only one direction. Note also that in the later case of asymmetric perturbation the resulting values of the collective diffusivities are very close, $D_{y y}^{c} / D_{z z}^{c} \sim 1$, while in the former case of the symmetric initial distortion this ratio is close to that from the DSF technique: $D_{y y M A}^{c} / D_{z z M A}^{c} \simeq 1.3$ and $D_{y y}^{c} / D_{z z}^{c} \simeq 1.4$. The better qualitative agreement with the results of the DSF approach here is not surprising: the relaxation of the particle density occurs simultaneously in the $y$ - and $z$-directions in the same way as the relaxation of the random density fluctuation in a steadily sheared suspension as probed by the DSF approach.

\section{Summary and concluding remarks}

In the present study we determined the shear-induced diffusivities (self- and gradient) using the dynamic structure factor (DSF) approach from Accelerated Stokesian Dynamics simulations. Monodisperse suspensions of non-colloidal hard spheres in a simple shear flow at zero Reynolds number were used in simulations. All calculations were performed in a steadily sheared suspension starting from initial random hard-sphere configurations after they have been pre-equilibrated for $20 \dot{\gamma} t$ strains. Using the dynamic structure factor approach we probed the time autocorrelations in particle number density while the shear-induced self- and gradient diffusivities are evaluated from the time rate of decay of appropriate dynamic structure factors. The expressions (2.12)-(2.13) are the appropriate generalization of the DSF approach that would allow the study of particle transport along an arbitrary direction in suspensions in shearing flows. In this work we only considered diffusion in the plane orthogonal to the flow, i.e. in the velocity-gradient and the vorticity directions.

For the self-diffusivity the leading term in the low- $k$ expansion of the self-dynamic structure factor is the integral of the velocity autocorrelation function, which is 
a well-known kinematic description of self-diffusivity. The self-diffusion coefficients evaluated from the DSF approach are in excellent agreement with previously reported results determined numerically from the time evolution of particles' mean-square displacements in the range of volume fractions $\phi=0.10-0.50$ (Sierou \& Brady 2004). Moreover, beyond $\phi=0.50$ when the shear-induced string-ordering transition takes place, an order-of-magnitude drop in the value of self-diffusivities along both transverse directions is observed. At a higher concentration of $\phi=0.60$, when the suspension is shear-melted to a disordered state, the values of both self-diffusivities increase abruptly to the maximum value observed, e.g. $D_{y y}^{s} \simeq 0.15 a^{2} \dot{\gamma}$.

For the gradient diffusivity, although the leading term in the low- $k$ expansion of the total dynamic structure factor leads to the appropriate kinematic description in terms of the integral of the velocity cross-correlation function and the static structure factor, higher-order terms in $k$ cannot be neglected even for systems of $N \sim 10^{3}$ particles. Therefore, the decay of the total dynamic structure factor is probed and the results are extrapolated to the limit of infinite box size to extract the gradient diffusivity corresponding to the limit $k \rightarrow 0$. The overall agreement between the results of the DSF approach and experiments is very good, although it should be noted that none of the experiments measure the gradient diffusivity directly; rather it is extracted from fitting particle migration data to the solution of model equations. To the best of the authors' knowledge, no dynamic scattering experiments that study diffusion in steadily sheared suspensions have been reported to date. Also, the present theory suggests that an approximate model for the gradient diffusivity is $\boldsymbol{D}^{\nabla} \sim \boldsymbol{D}^{s} / S^{e q}(0)$, which agrees qualitatively well with the full results of the DSF approach and the available experimental measurements for a wide range of particle volume fractions.

It was also found that the values of the shear-induced gradient diffusivity reported earlier by Marchioro \& Acrivos (2001) are significantly underestimated due to the small system size available with conventional SD simulations. Although there is a good agreement between results based on relaxation of the dynamic structure factor and those of Marchioro \& Acrivos (2001) (after being correctly extrapolated to the limit of infinite system size), we showed that their approach involves relaxation of spatial inhomogeneities of particle density and is, therefore, rather sensitive to the amplitude and the shape of the initial distortion of the suspension microstructure.

The major advantage of the proposed approach is that the simulations are performed on statistically homogeneous, steadily sheared suspensions, without introducing any artificial perturbations to the suspension microstructure. This also allows us to determine both diffusivities (self- and the gradient) in both transverse directions from the same series of simulations. Furthermore, the DSF approach does not depend on particle-level dynamics and therefore is the natural starting point for determining the diffusivity in any system whether in equilibrium or not.

The authors would like to thank Ileana Carpen for providing results of the Brownian dynamics simulations. A. M. L. also acknowledges the support of the Lester Deutch Foundation.

\section{REFERENCES}

ACKerson, B. J. \& Pusey, P. N. 1988 Shear-induced order in suspensions of hard-spheres. Phys. Rev. Lett. 61, 1033-1036.

Acrivos, A., Batchelor, G. K., Hinch, E. J., Koch, D. L. \& Mauri, R. 1992 The longitudinal shear-induced diffusion of spheres in a dilute suspension. J. Fluid Mech. 240, 651-657. 
BAtCHelor, G. K. 1976 Brownian diffusion of particles with hydrodynamic interaction. J. Fluid Mech. 74, 1-29.

Berne, B. J. \& Pecora, R. 1976 Dynamic Light Scattering. Wiley.

Bossis, G. \& Brady, J. F. 1984 Dynamic simulation of sheared suspensions. I. General method. J. Chem. Phys. 80, 5141-5154.

Brady, J. F. \& Morris, J. F. 1997 Microstructure of strongly sheared suspensions and its impact on rheology and diffusion. J. Fluid Mech. 348, 103-139.

Breedveld, V., Van den Ende, D., Bosscher, M., Jongschaap, R. J. J. \& Mellema, J. 2001 Measuring shear-induced self-diffusion in a counter-rotating geometry. Phys. Rev. E 63, $1403-1412$.

Breedveld, V., Van den Ende, D., Triphati, A. \& Acrivos, A. 1998 The measurement of the shear-induced particle and fluid tracer diffusivities by a novel method. J. Fluid Mech. 375, 297-318.

Chen, L. B., Zukoski, C. F., Ackerson, B. J., Hanley, H. J. M., Straty, G. C., Barker, J. \& Glinka, C. J. 1992 Structural changes and orientational order in a sheared colloidal suspension. Phys. Rev. Lett. 69, 688-691.

Da Cunha, F. R. \& Hinch, E. J. 1996 Shear-induced disperion in a dilute suspesnion of rough spheres. J. Fluid Mech. 309, 211-223.

Drazer, G., Koplik, J., Khusid, B. \& Acrivos, A. 2002 Deterministic and stochastic behavior of non-Brownian spheres in sheared suspensions. J. Fluid Mech. 460, 307-335.

Eckstein, E. C., Bailey, D. G. \& Shapiro, A. H. 1977 Self-diffusion of particles in shear-flow of a suspension. J. Fluid Mech. 79, 191-208.

ElRick, D. E. 1962 Source functions for diffusion in uniform shear flow. Austral. J. Phys. 15, 283-288.

ERPENBECK, J. J. 1984 Shear viscosity of the hard-sphere fluid via nonequilibrium moleculardynamics. Phys. Rev. Lett. 52, 1333-1335.

Evans, D. J. \& Morriss, G. P. 1986 Shear thickening and turbulence in simple fluids. Phys. Rev. Lett. 56, 2172-2175.

Foss, D. R. \& BRAdY, J. F. 1999 Self-diffusion in sheared suspensions by dynamic simulation. J. Fluid Mech. 401, 243-274.

Gadala-Maria, F. \& ACRIVOs, A. 1980 Shear-induced structure in a concentrated suspension of solid spheres. J. Rheology 24, 799-814.

Hansen, J.-P. \& McDonald, I. R. 1986 Theory of Simple Liquids. Academic.

Jánosi, I. M., Tél, T., Wolf, D. E. \& Gallas, A. C. 1997 Chaotic particle dynamics in viscous flow: The three-particle Stokeslet problem. Phys. Rev. E 56, 2858-2868.

Johnson, S. J., DE Kruif, C. G. \& MAY, R. P. 1988 Structure factor distortion for hard-sphere dispersions subjected to weak shear flow: Small-angle neutron scattering in the flow-vorticity plane. J. Chem. Phys. 89, 5909-5921.

de Kruif, C. G., van der WerfF, J. C., Johnson, S. J. \& May, R. P. 1990 Small-angle neutron scattering of sheared concentrated dispersions: Microstructure along principal axes. Phys. Fluids 2, 1545-1556.

LeaL, L. G. 1973 On the effective conductivity of a dilute suspension of spherical drops in the limit of low Péclet number. Chem. Engng Commun. 1, 21.

Leighton, D. \& Acrivos, A. 1987 The shear-induced migration of particles in concentrated suspensions. J. Fluid Mech. 181, 415-439.

Marchioro, M. \& ACrivos, A. 2001 Shear-induced particle diffusivities from numerical simuations. J. Fluid Mech. 443, 101-128.

Morris, J. F. \& Brady, J. F. 1996 Self-diffusion in sheared suspensions. J. Fluid Mech. 312, 223-252.

Nir, A. \& ACrivos, A. 1976 The effective conductivity of sheared suspensions. J. Fluid Mech. 78, 33-48.

Phillips, R. J., Armstrong, R. C., Brown, R. A., Graham, A. L. \& Abbot, J. R. 1992 A constitutive equation for concentrated suspensions that accounts for shear-induced particle migration. Phys. Fluids A 4, 30-40.

Pusey, P. N. 1991 Colloidal suspensions. In Liquids, Freezing and Glass Transition (ed. J. P. Hansen, D. Levesque \& J. Zinn-Justin). Elsevier.

Rallison, J. M. \& HinCH, E. J. 1986 The effect of particle interactions on dynamic light scattering from a dilute suspension. J. Fluid Mech. 167, 131-168. 
Russel, W. B. \& Glendinning, A. B. 1981 The effective diffusion coefficient detected by dynamic light scattering. J. Chem. Phys. 72, 948-952.

Segré, P. N., Behrend, O. P. \& Pusey, P. N. 1995 Short-time Brownian motion in colloidal suspenions: Experiment and simulation. Phys. Rev. E 52, 5070-5083.

Sierou, A. \& Brady, J. F. 2001 Accelerated Stokesian Dynamics Simulations. J. Fluid Mech. 448, $115-146$.

Sierou, A. \& Brady, J. F. 2002 Rheology and microstructure in concentrated noncolloidal suspensions. J. Rheol. 46, 1031-1056.

Sierou, A. \& Brady, J. F. 2004 Shear-induced self-diffusion in non-colloidal suspensions. J. Fluid Mech. 506, 285-314.

WAGNeR, N. J. \& Russel, W. B. 1990 Light scattering experiements of a hard-sphere suspension under shear. Phys. Fluids A 2, 491-502.

WANG, Y., MAURI, R. \& ACrivos, A. 1996 The transverse shear-induced liquid and particle tracer diffusivities of a dilute suspension of spheres undergoing a simple shear flow. J. Fluid Mech. 327, 255-272.

WANG, Y., Mauri, R. \& ACrivos, A. 1998 Transverse shear-induced gradient diffusivition of a dilute suspension of spheres. J. Fluid Mech. 357, 279-287.

Xue, W. \& Grest, G. S. 1990 Shear-induced alighnment of colloidal particles in the presence of a shear flow. Phys. Rev. Lett. 64, 419-422. 